\title{
A Table Lookup Method for Exact Analytical Solutions of Nonlinear Fractional Partial Differential Equations
}

\author{
Ji Juan-Juan, ${ }^{1,2}$ Guo Ye-Cai, ${ }^{3}$ Zhang Lan-Fang, ${ }^{2}$ and Zhang Chao-Long ${ }^{2}$ \\ ${ }^{1}$ College of Atmospheric Science, Nanjing University of Information Science \& Technology, Nanjing 210044, China \\ ${ }^{2}$ School of Physics and Electronic Engineering, Anqing Normal University, Anqing 246133, China \\ ${ }^{3}$ School of Electronic \& Information Engineering, Nanjing University of Information Science \& Technology, Nanjing 210044, China
}

Correspondence should be addressed to Ji Juan-Juan; jjj0721@126.com

Received 11 August 2016; Accepted 3 November 2016; Published 3 January 2017

Academic Editor: Hang Xu

Copyright (C) 2017 Ji Juan-Juan et al. This is an open access article distributed under the Creative Commons Attribution License, which permits unrestricted use, distribution, and reproduction in any medium, provided the original work is properly cited.

\begin{abstract}
A table lookup method for solving nonlinear fractional partial differential equations (fPDEs) is proposed in this paper. Looking up the corresponding tables, we can quickly obtain the exact analytical solutions of fPDEs by using this method. To illustrate the validity of the method, we apply it to construct the exact analytical solutions of four nonlinear fPDEs, namely, the time fractional simplified $\mathrm{MCH}$ equation, the space-time fractional combined KdV-mKdV equation, the $(2+1)$-dimensional time fractional Zoomeron equation, and the space-time fractional ZKBBM equation. As a result, many new types of exact analytical solutions are obtained including triangular periodic solution, hyperbolic function solution, singular solution, multiple solitary wave solution, and Jacobi elliptic function solution.
\end{abstract}

\section{Introduction}

Fractional partial differential equations (fPDEs) are the generalized form of the integer order differential equations. fPDEs can more accurately describe the complex physical phenomena occurring in fluid dynamics, high-energy physics, plasma physics, elastic media, optical fibers, chemical kinematics, chemical physics, acoustic waves, biomathematics, and many other areas $[1,2]$. In recent years, many researchers have shown great interest in the search for exact solutions to nonlinear fPDEs. At present, several methods for finding the exact solutions of fPDEs have been presented, for example, the Adomian decomposition method [3-6], variational iteration method [7-9], homotopy perturbation method [10-13], homotopy analysis method [14, 15], differential transform method [16], spectral methods $[17,18]$, discontinuous Galerkin method [19], Kansa method [20], fractional subequation method [21], generalized fractional subequation method [22], fractional projective Riccati expansion method [23], exp-function method [24, 25], $\left(G^{\prime} / G\right)$-expansion method [26-28], functional variable method $[29,30]$, and first integral method $[31,32]$.

However, the above methods either are relatively complicated or have large computational cost. We propose a table lookup method in this paper. This method is straightforward and has small computational cost. We apply it to solve nonlinear fractional order partial differential equations with using the fractional complex transform and the modified RiemannLiouville derivative defined by Jumarie [33]. Jumarie's modified Riemann-Liouville derivative of order $\alpha$ is defined by the following expression [34]:

$$
D_{x}^{\alpha} f(x)= \begin{cases}\frac{1}{\Gamma(1-\alpha)} \int_{0}^{x}(x-\xi)^{-\alpha-1}[f(\xi)-f(0)] d \xi & \alpha<0, \\ \frac{1}{\Gamma(1-\alpha)} \frac{d}{d x} \int_{0}^{x}(x-\xi)^{-\alpha}[f(\xi)-f(0)] d \xi & 0<\alpha<1, \\ {\left[f^{(\alpha-n)}(x)\right]^{(n)},} & n \leq \alpha<n+1, n \geq 1,\end{cases}
$$


where $f(x)$ denotes a continuous function and $\Gamma(\cdot)$ is the Gamma function.

Moreover, the modified Riemann-Liouville derivative has various useful properties, including the following:

$$
\begin{aligned}
D_{x}^{\alpha} x^{\gamma} & =\frac{\Gamma(\gamma+1)}{\Gamma(\gamma+1-\alpha)} x^{\gamma-\alpha}, \quad \gamma>0 \\
D_{x}^{\alpha}(c f(x)) & =c D_{x}^{\alpha} f(x), \\
D_{x}^{\alpha}(f(x) g(x)) & =g(x) D_{x}^{\alpha} f(x)+f(x) D_{x}^{\alpha} g(x), \\
D_{x}^{\alpha} f(x)[g(x)] & =f_{g}^{\prime}[g(x)] D_{x}^{\alpha} g(x) \\
& =D_{g}^{\alpha}[g(x)]\left(g_{x}^{\prime}\right)^{\alpha} .
\end{aligned}
$$

The rest of this paper is organized as follows. In Section 2, the basic ideas and main steps of the table lookup method are given. In Section 3, the exact analytical solutions of the time fractional simplified $\mathrm{MCH}$ equation, the space-time combined KdV-mKdV equation, the $(2+1)$-dimensional time fractional Zoomeron equation, and the space-time fractional ZKBBM equation are constructed using the proposed method. In Section 4, some conclusions are obtained.

\section{Basic Idea of the Table Lookup Method}

In this section, we outline the main steps of the table lookup method for solving nonlinear fPDEs. Let us consider a fractional order partial differential equation in the following form:

$$
\begin{aligned}
H\left(u, D_{t}^{\alpha} u, D_{x}^{\beta} u, D_{y}^{\gamma} u, D_{t}^{\alpha} D_{t}^{\alpha} u, D_{t}^{\alpha} D_{x}^{\beta} u, \ldots\right)= & 0, \\
& 0<\alpha, \beta,
\end{aligned}
$$

where $D_{t}^{\alpha} u, D_{x}^{\beta} u$, and $D_{y}^{\gamma} u$ denote modified RiemannLiouville derivatives of $u(x, y, t), H$ represents a polynomial in $u(x, y, t)$ and its various partial derivatives, and $x, y$, and $t$ are variables. In the following, we give the main steps of our proposed method.

Step 1. First, we use the fractional complex transformation as follows:

$$
\begin{aligned}
& u(x, y, t)=u(\xi) \\
& \xi \xi=\frac{y^{\gamma}}{\Gamma(1+\gamma)}+\frac{x^{\beta}}{\Gamma(1+\beta)}-\frac{c t^{\alpha}}{\Gamma(1+\alpha)},
\end{aligned}
$$

where $c$ is a nonzero constant. Thus, (6) can be transformed into the following nonlinear ordinary differential equation (ODE) of integer order with respect to $\xi$ in sense of the properties of Jumarie's modified Riemann-Liouville derivative given in (2)-(5):

$$
\widetilde{H}\left(u, u^{\prime}, u^{\prime \prime}, u^{\prime \prime \prime}, \ldots\right)=0
$$

where $\widetilde{H}$ is a polynomial in $u(\xi)$ and its various derivatives $u^{\prime}, u^{\prime \prime}, u^{\prime \prime \prime}, \ldots$ and $u^{\prime}=d u / d \xi, u^{\prime \prime}=d^{2} u / d \xi^{2}, \ldots$
Step 2. Integrating (8) once or several times with respect to $\xi$, setting the integration constant to zero if possible, multiplying both sides of the equation by $u^{\prime}$, and then integrating once again, we obtain different types of the auxiliary equation $\left(\varphi^{\prime}\right)^{2}=a_{0}+a_{1} \varphi+a_{2} \varphi^{2}+a_{3} \varphi^{3}+a_{4} \varphi^{4}$, where $a_{i}(i=0,1, \ldots, 4)$ are constant coefficients that can be determined in this step.

Step 3. According to the equation type obtained in Step 2, we create the tables of solutions to the corresponding type of auxiliary equation.

Step 4. Looking up the tables and determining the corresponding coefficients and existence conditions of the exact solutions, we successfully obtain the exact analytical solutions to $(6)$.

\section{Applications of the Proposed Method}

In this section, we apply the table lookup method to construct exact analytical solutions of four nonlinear fPDEs, namely, the time fractional simplified $\mathrm{MCH}$ equation, the space-time combined $\mathrm{KdV}$-MKdV equation, the $(2+1)$-dimensional time fractional Zoomeron equation, and the space-time fractional ZKBBM equation, which are very important nonlinear fPDEs in mathematical physics and have received much attention from researchers.

3.1. The Time Fractional Simplified Modified Camassa-Holm $(\mathrm{MCH})$ Equation. We now consider the following time fractional simplified modified Camassa-Holm $(\mathrm{MCH})$ equation:

$$
D_{t}^{\alpha} u+2 k u_{x}-D_{t}^{\alpha}\left(u_{x x}\right)+\beta u^{2} u_{x}=0
$$

where $k \in \mathfrak{R}, \beta>0$, and $0<\alpha<1$. Equation (9) is a variation of the following equation:

$$
u_{t}+2 k u_{x}-u_{x x t}+\beta u^{2} u_{x}=0 .
$$

Using the fractional complex transformation $u(x, t)=$ $u(\xi), \xi=x-c t^{\alpha} / \Gamma(1+\alpha)$ with (9), we can obtain the following nonlinear ODE:

$$
-c u^{\prime}+2 k u^{\prime}+c u^{\prime \prime \prime}+\beta u^{2} u^{\prime}=0 .
$$

Integrating (11) once with respect to $\xi$ and setting the integral constant to zero, we obtain

$$
(2 k-c) u+c u^{\prime \prime}+\frac{\beta}{3} u^{3}=0
$$

Multiplying (12) by $u^{\prime}$ and then integrating once again, we obtain

$$
\left(u^{\prime}\right)^{2}=a_{0}+a_{2} u^{2}+a_{4} u^{4}
$$

where $a_{2}=-(2 k-c) / c, a_{4}=-\beta / 6 c$, and $a_{0}$ is an integration constant.

According to the solutions of the auxiliary equation presented in [35], the exact solutions of (14) are listed in Table 1:

$$
\left(\varphi^{\prime}\right)^{2}=a_{0}+a_{2} \varphi^{2}+a_{4} \varphi^{4}
$$


TABLE 1: Solutions of (14), $\varepsilon= \pm 1$.

$$
\begin{aligned}
& \text { Number } \varphi
\end{aligned}
$$

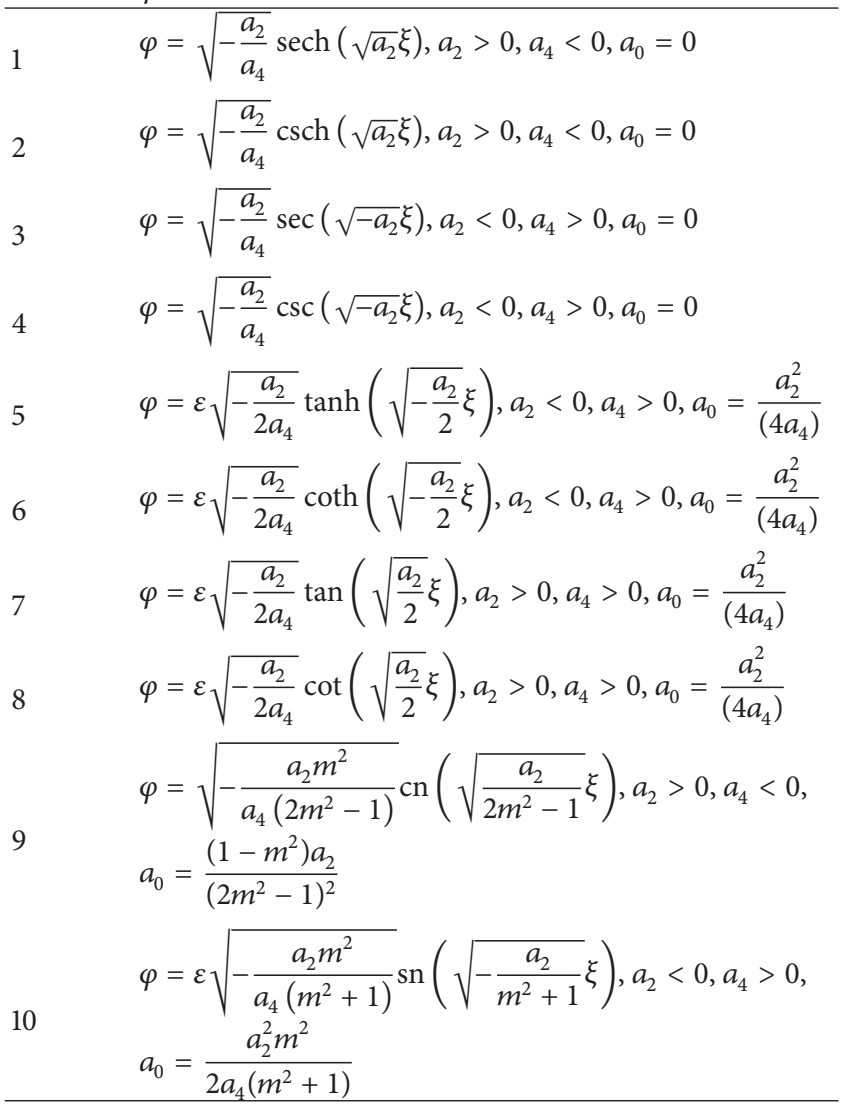

Equations (13) and (14) have the same form. Thus, looking up Table 1 and determining the coefficients and the existence condition of the exact solutions, we can obtain the following solutions of (9):

(1) When $c>2 k \& c>0, a_{0}=0$, and $\varepsilon= \pm 1$, we obtain

$$
\begin{aligned}
& u_{1}(x, t)=\sqrt{-\frac{6(2 k-c)}{\beta}} \\
& \cdot \operatorname{sech}\left(\sqrt{-\frac{2 k-c}{c}}\left(x-\frac{c t^{\alpha}}{\Gamma(1+\alpha)}\right)\right), \\
& u_{2}(x, t)=\sqrt{-\frac{6(2 k-c)}{\beta}} \\
& \cdot \operatorname{csch}\left(\sqrt{-\frac{2 k-c}{c}}\left(x-\frac{c t^{\alpha}}{\Gamma(1+\alpha)}\right)\right) .
\end{aligned}
$$

(2) When $2 k<c<0, a_{0}=0$, and $\varepsilon= \pm 1$, we obtain $u_{3}(x, t)$

$$
=\sqrt{-\frac{6(2 k-c)}{\beta}} \sec \left(\sqrt{\frac{2 k-c}{c}}\left(x-\frac{c t^{\alpha}}{\Gamma(1+\alpha)}\right)\right),
$$

$u_{4}(x, t)$

$$
=\sqrt{-\frac{6(2 k-c)}{\beta}} \csc \left(\sqrt{\frac{2 k-c}{c}}\left(x-\frac{c t^{\alpha}}{\Gamma(1+\alpha)}\right)\right) .
$$

(3) When $2 k<c<0, a_{0}=-3(2 k-c)^{2} / 2 \beta c$, and $\varepsilon= \pm 1$, we obtain

$$
\begin{aligned}
& u_{5}(x, t)=\varepsilon \sqrt{-\frac{3(2 k-c)}{\beta}} \\
& \cdot \tanh \left(\sqrt{\frac{2 k-c}{2 c}}\left(x-\frac{c t^{\alpha}}{\Gamma(1+\alpha)}\right)\right), \\
& u_{6}(x, t)=\varepsilon \sqrt{-\frac{3(2 k-c)}{\beta}} \\
& \cdot \operatorname{coth}\left(\sqrt{\frac{2 k-c}{2 c}}\left(x-\frac{c t^{\alpha}}{\Gamma(1+\alpha)}\right)\right) .
\end{aligned}
$$

(4) When $c<2 k \& c<0, a_{0}=-3(2 k-c)^{2} / 2 \beta c$, and $\varepsilon= \pm 1$, we obtain $u_{7}(x, t)$

$$
=\varepsilon \sqrt{\frac{3(2 k-c)}{\beta}} \tan \left(\sqrt{-\frac{2 k-c}{2 c}}\left(x-\frac{c t^{\alpha}}{\Gamma(1+\alpha)}\right)\right),
$$

$u_{8}(x, t)$

$$
=\varepsilon \sqrt{\frac{3(2 k-c)}{\beta}} \cot \left(\sqrt{-\frac{2 k-c}{2 c}}\left(x-\frac{c t^{\alpha}}{\Gamma(1+\alpha)}\right)\right) .
$$

(5) When $c>2 k \& c>0, a_{0}=-(2 k-c)\left(1-m^{2}\right) / c\left(2 m^{2}-\right.$ $1)^{2}$, and $\varepsilon= \pm 1$, we obtain

$$
\begin{aligned}
& u_{9}(x, t)=\sqrt{-\frac{6(2 k-c) m^{2}}{\beta\left(2 m^{2}-1\right)}} \\
& \cdot \operatorname{cn}\left(\sqrt{-\frac{2 k-c}{2 c\left(2 m^{2}-1\right)}}\left(x-\frac{c t^{\alpha}}{\Gamma(1+\alpha)}\right)\right) .
\end{aligned}
$$

(6) When $c>2 k \& c<0, a_{0}=-3(2 k-c)^{2} m^{2} / 2 \beta c\left(m^{2}+\right.$ 1 ), and $\varepsilon= \pm 1$, we obtain

$$
\begin{aligned}
u_{10}(x, t)= & \varepsilon \sqrt{-\frac{6(2 k-c) m^{2}}{\beta\left(m^{2}+1\right)}} \\
& \cdot \operatorname{sn}\left(\sqrt{\frac{2 k-c}{c\left(m^{2}+1\right)}}\left(x-\frac{c t^{\alpha}}{\Gamma(1+\alpha)}\right)\right) .
\end{aligned}
$$


The evolution of exact solution for (15)-(24) is shown in Figures 1-6.

It can be observed from (15)-(24) and Figures 1-6 that triangular periodic solution, bell-shaped solitary wave solution, kink-shaped solitary wave solution, and Jacobi elliptic function solution of the time fractional simplified $\mathrm{MCH}$ are obtained.

When $\alpha=1$, (9) becomes (10), namely, simplified MCH equation, and $\xi=x-c t$, obviously, (10) still have triangular periodic solution, bell-shaped solitary wave solution, kinkshaped solitary wave solution, and Jacobi elliptic function four types of solutions. Reference [36] only obtained triangular periodic solutions, kink-shaped solitary wave solution, and singular solution of simplified $\mathrm{MCH}$ equation. Thus, our table lookup method is more powerful.

3.2. The Space-Time Fractional Combined KdV-mKdV Equation. In this section, we will apply the table lookup method to the following space-time fractional combined KdV-mKdV equation [37]:

$$
D_{t}^{\alpha} u+\mu u D_{x}^{\alpha} u+\delta u^{2} D_{x}^{\alpha} u+D_{x}^{3 \alpha} u=0
$$

where $\mu$ and $\delta$ are nonzero constants. This equation may describe the wave propagation of a bound particle, sound wave, or thermal pulse.

Now, we use the fractional complex transformation $u(x, t)=u(\xi), \xi=x^{\alpha} / \Gamma(1+\alpha)-c\left(t^{\alpha} / \Gamma(1+\alpha)\right)$ with (25), which is reduced to the following ODE of integer order:

$$
-c u^{\prime}+\mu u u^{\prime}+\delta u^{2} u^{\prime}+u^{\prime \prime \prime}=0 .
$$

Integrating (26) once with the integral constant of zero yields

$$
-c u+\frac{\mu}{2} u^{2}+\frac{\delta}{3} u^{3}+u^{\prime \prime}=0
$$

Multiplying (27) by $u^{\prime}$, integrating once again, and setting the integral constant to zero, we obtain

$$
\left(u^{\prime}\right)^{2}=a_{2} u^{2}+a_{3} u^{3}+a_{4} u^{4}
$$

where $a_{2}=c, a_{3}=-\mu / 3$, and $a_{4}=-\delta / 6$.

By virtue of the solutions of the auxiliary equation given in [38], the exact solutions of (29) are shown in Table 2:

$$
\left(\varphi^{\prime}\right)^{2}=a_{2} \varphi^{2}+a_{3} \varphi^{3}+a_{4} \varphi^{4}
$$

Equations (28) and (29) have the same form. Thus, looking up Table 2 and determining the coefficients and existence conditions of the exact solutions, we can obtain the following solutions of (25):

(1) If $c>0$ and $\varepsilon= \pm 1$, we obtain

$$
\begin{aligned}
& u_{1}(x, t)=\frac{(c \mu / 3) \operatorname{sech}^{2}\left((\sqrt{c} / 2)\left(x^{\alpha} / \Gamma(1+\alpha)-c t^{\alpha} / \Gamma(1+\alpha)\right)\right)}{\mu / 3+(\delta c / 6)\left(1+\varepsilon \tanh \left((\sqrt{c} / 2)\left(x^{\alpha} / \Gamma(1+\alpha)-c t^{\alpha} / \Gamma(1+\alpha)\right)\right)\right)^{2}}, \\
& u_{2}(x, t)=\frac{(c \mu / 3) \operatorname{csch}^{2}\left((\sqrt{c} / 2)\left(x^{\alpha} / \Gamma(1+\alpha)-c t^{\alpha} / \Gamma(1+\alpha)\right)\right)}{\mu / 3+(\delta c / 6)\left(1+\varepsilon \operatorname{coth}\left((\sqrt{c} / 2)\left(x^{\alpha} / \Gamma(1+\alpha)-c t^{\alpha} / \Gamma(1+\alpha)\right)\right)\right)^{2}} .
\end{aligned}
$$

(2) If $c>0, \mu^{2}>-6 \delta c$, and $\varepsilon= \pm 1$, we obtain

$$
\begin{aligned}
& u_{3}(x, t) \\
& =\frac{6 c \operatorname{sech}\left(\sqrt{c}\left(x^{\alpha} / \Gamma(1+\alpha)-c t^{\alpha} / \Gamma(1+\alpha)\right)\right)}{\varepsilon \sqrt{\mu^{2}+6 \delta c}+\mu \operatorname{sech}\left(\sqrt{c}\left(x^{\alpha} / \Gamma(1+\alpha)-c t^{\alpha} / \Gamma(1+\alpha)\right)\right)} .
\end{aligned}
$$

(3) If $c<0, \mu^{2}>-6 \delta c$, and $\varepsilon= \pm 1$, we obtain

$$
\begin{aligned}
& u_{4}(x, t) \\
& =\frac{6 c \sec \left(\sqrt{-c}\left(x^{\alpha} / \Gamma(1+\alpha)-c t^{\alpha} / \Gamma(1+\alpha)\right)\right)}{\varepsilon \sqrt{\mu^{2}+6 \delta c}+\mu \sec \left(\sqrt{-c}\left(x^{\alpha} / \Gamma(1+\alpha)-c t^{\alpha} / \Gamma(1+\alpha)\right)\right)},
\end{aligned}
$$

$$
\begin{aligned}
& u_{5}(x, t) \\
& =\frac{6 c \csc \left(\sqrt{-c}\left(x^{\alpha} / \Gamma(1+\alpha)-c t^{\alpha} / \Gamma(1+\alpha)\right)\right)}{\varepsilon \sqrt{\mu^{2}+6 \delta c}+\mu \csc \left(\sqrt{-c}\left(x^{\alpha} / \Gamma(1+\alpha)-c t^{\alpha} / \Gamma(1+\alpha)\right)\right)} .
\end{aligned}
$$

(4) If $c>0, \mu^{2}<-6 \delta c$, and $\varepsilon= \pm 1$, we obtain

$$
\begin{aligned}
& u_{6}(x, t) \\
& =\frac{6 c \operatorname{csch}\left(\sqrt{c}\left(x^{\alpha} / \Gamma(1+\alpha)-c t^{\alpha} / \Gamma(1+\alpha)\right)\right)}{\varepsilon \sqrt{-\left(\mu^{2}+6 \delta c\right)}+\mu \operatorname{csch}\left(\sqrt{c}\left(x^{\alpha} / \Gamma(1+\alpha)-c t^{\alpha} / \Gamma(1+\alpha)\right)\right)} .
\end{aligned}
$$



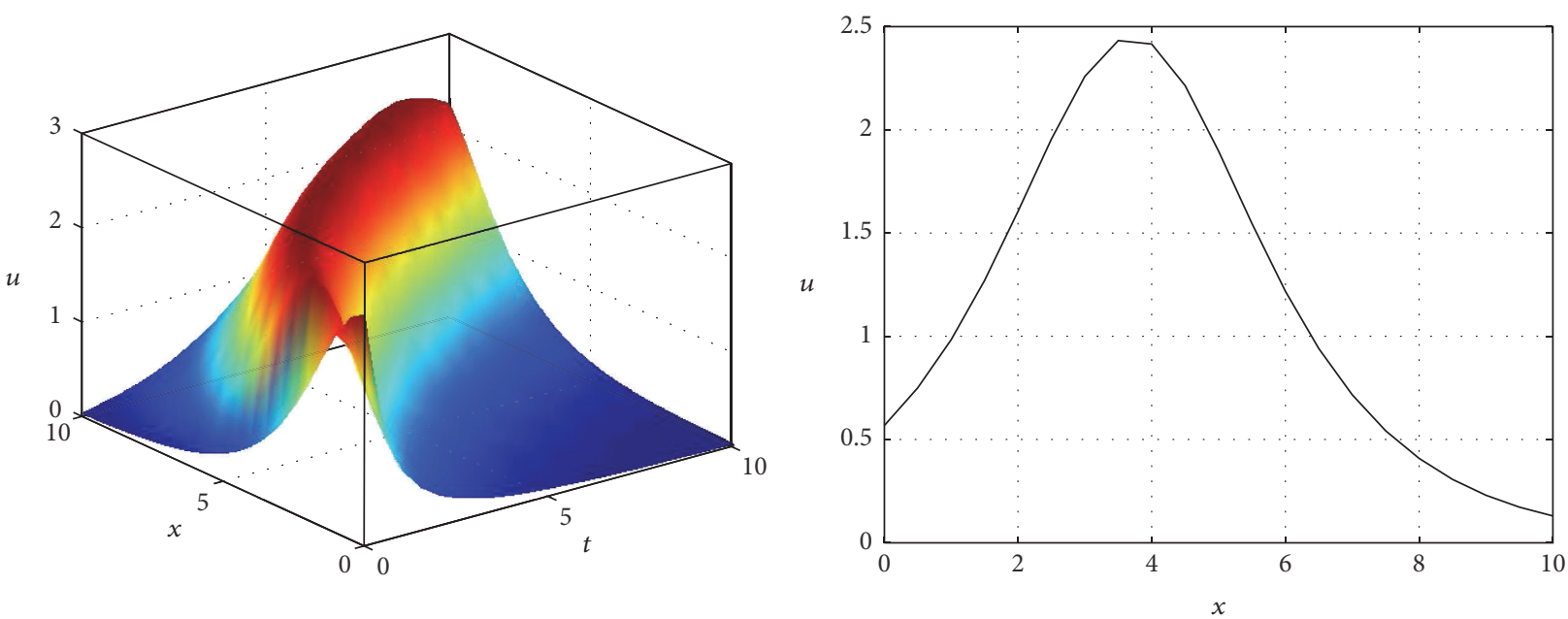

FIGURE 1: Exact solutions $u_{1}(x, t)$ for (15) and its position at $t=1.5$, when the parameters $k=1, \beta=1, c=3$, and $\alpha=0.5$.
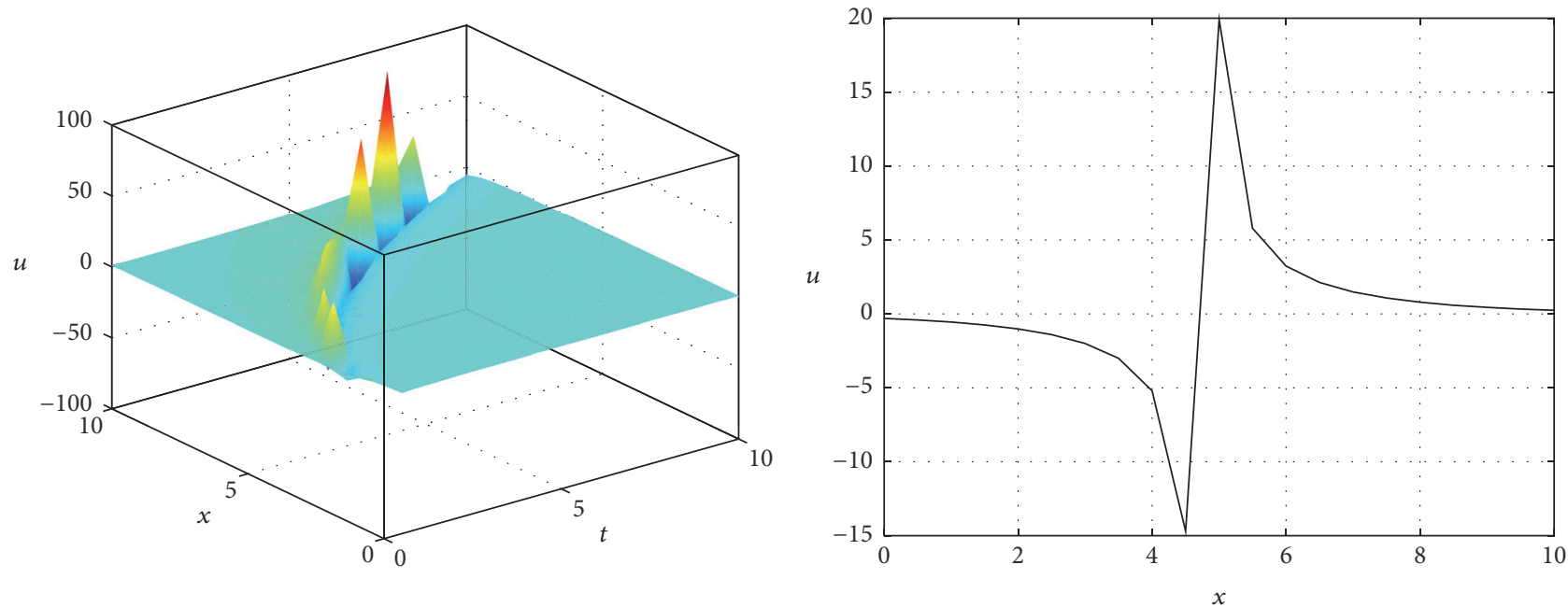

FIgURE 2: Exact solutions $u_{2}(x, t)$ for (16) and its position at $t=2$, when the parameters $k=1, \beta=1, c=3$, and $\alpha=0.5$.
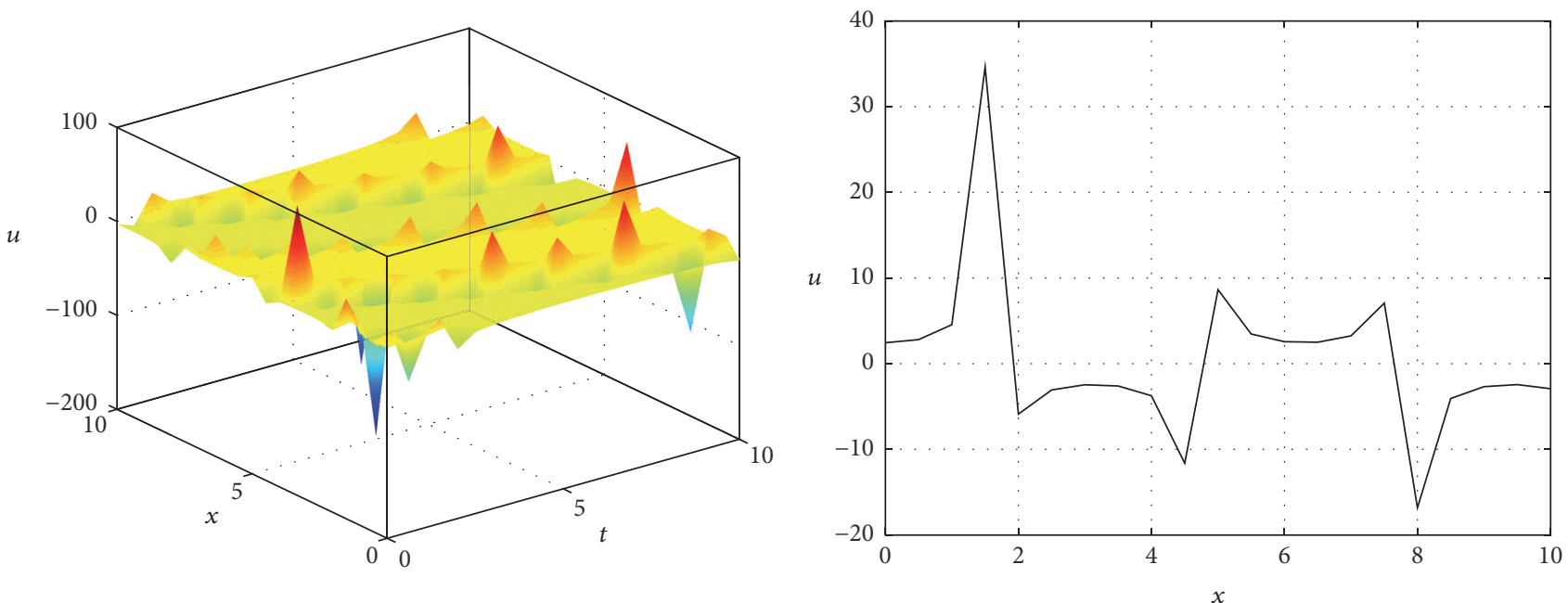

FIGURE 3: Exact solutions $u_{3}(x, t)$ for (17) and its position at $t=0$, when the parameters $k=-1, \beta=1, c=-1$, and $\alpha=0.6$. 

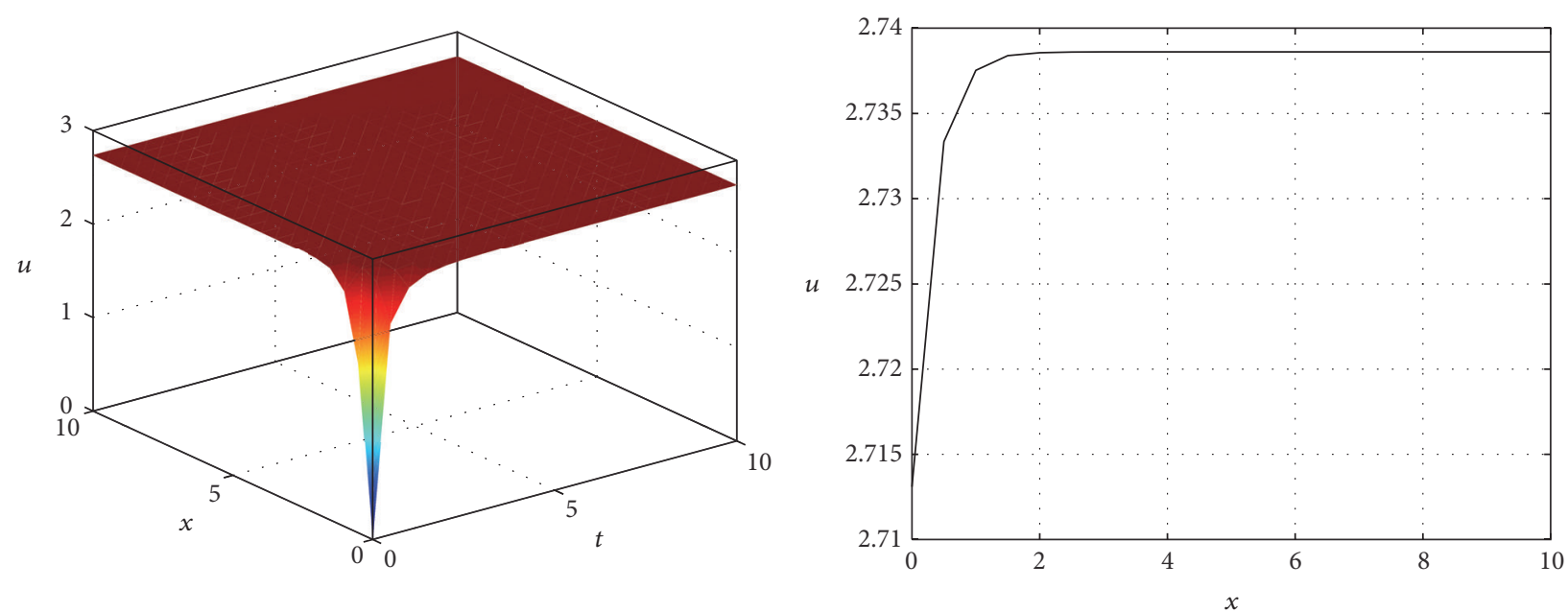

FIGURE 4: Exact solutions $u_{5}(x, t)$ for (19) and its position at $t=2$, when the parameters $k=-3, \beta=2, c=-1, \varepsilon=1$, and $\alpha=0.6$.
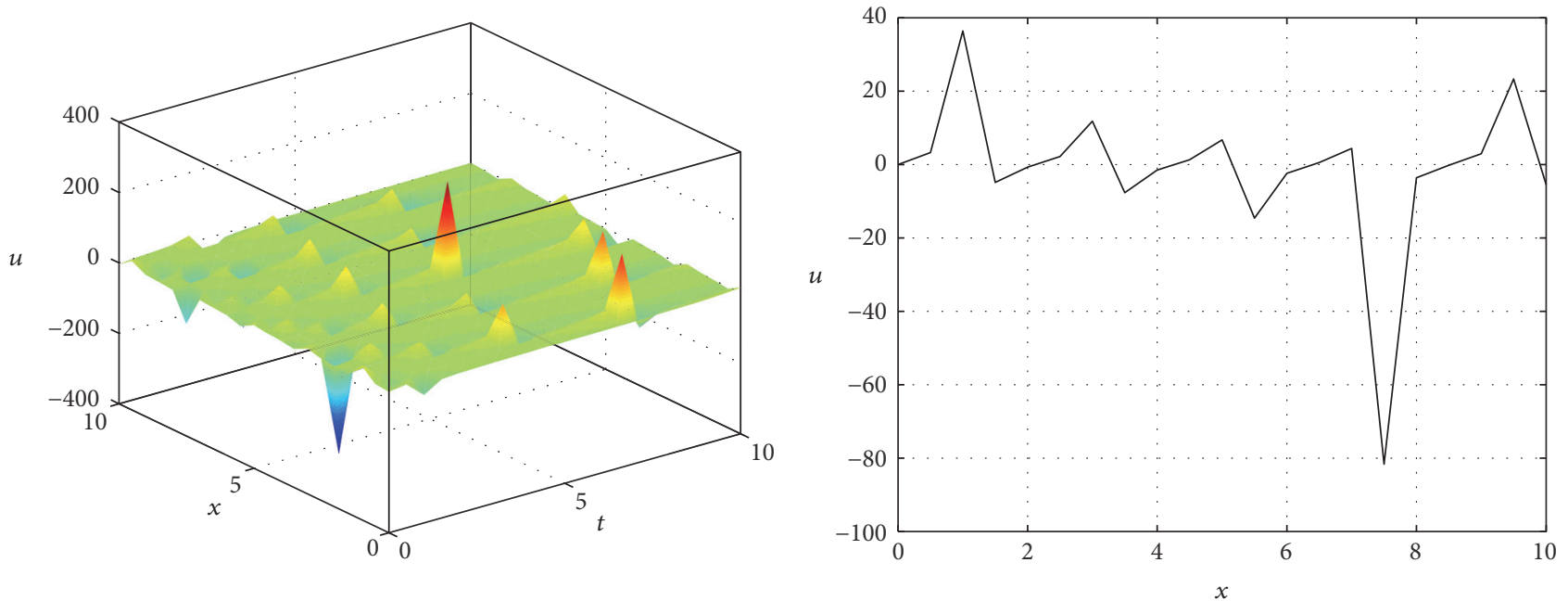

FIGURE 5: Exact solutions $u_{7}(x, t)$ for (21) and its position at $t=0$, when the parameters $k=5, \beta=3, c=-3$, and $\alpha=0.2$.
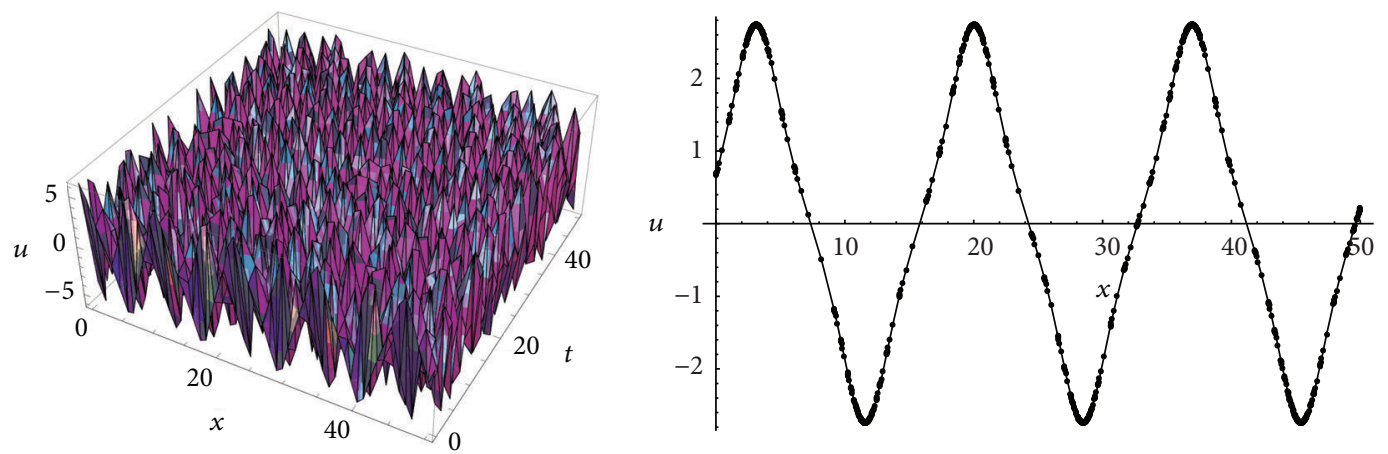

FIGURE 6: Exact solutions $u_{9}(x, t)$ for $(23)$ and its position at $t=0$, when the parameters $k=1, \beta=1.2, c=7$, and $\alpha=0.8$. 
(5) If $c>0, \delta<0$, and $\varepsilon= \pm 1$, we obtain

$$
\begin{aligned}
& u_{7}(x, t)=\frac{-c \operatorname{sech}^{2}\left((\sqrt{c} / 2)\left(x^{\alpha} / \Gamma(1+\alpha)-c t^{\alpha} / \Gamma(1+\alpha)\right)\right)}{-\mu / 3+2 \varepsilon \sqrt{-\delta c / 6} \tanh \left((\sqrt{c} / 2)\left(x^{\alpha} / \Gamma(1+\alpha)-c t^{\alpha} / \Gamma(1+\alpha)\right)\right)}, \\
& u_{8}(x, t)=\frac{(c \mu / 3) \operatorname{csch}^{2}\left((\sqrt{c} / 2)\left(x^{\alpha} / \Gamma(1+\alpha)-c t^{\alpha} / \Gamma(1+\alpha)\right)\right)}{\mu^{2} / 9+(\delta c / 6)\left(1+\varepsilon \operatorname{coth}\left((\sqrt{c} / 2)\left(x^{\alpha} / \Gamma(1+\alpha)-c t^{\alpha} / \Gamma(1+\alpha)\right)\right)\right)} .
\end{aligned}
$$

(6) If $c<0, \delta<0$, and $\varepsilon= \pm 1$, we obtain

$$
\begin{gathered}
u_{9}(x, t)=\frac{-c \sec ^{2}\left((\sqrt{-c} / 2)\left(x^{\alpha} / \Gamma(1+\alpha)-c t^{\alpha} / \Gamma(1+\alpha)\right)\right)}{-\mu / 3+2 \varepsilon \sqrt{\delta c / 6} \tan \left((\sqrt{-c} / 2)\left(x^{\alpha} / \Gamma(1+\alpha)-c t^{\alpha} / \Gamma(1+\alpha)\right)\right)}, \\
u_{10}(x, t)=\frac{-c \csc ^{2}\left((\sqrt{-c} / 2)\left(x^{\alpha} / \Gamma(1+\alpha)-c t^{\alpha} / \Gamma(1+\alpha)\right)\right)}{-\mu / 3+2 \varepsilon \sqrt{\delta c / 6} \cot \left((\sqrt{-c} / 2)\left(x^{\alpha} / \Gamma(1+\alpha)-c t^{\alpha} / \Gamma(1+\alpha)\right)\right)} .
\end{gathered}
$$

(7) If $c>0, \mu^{2}=-6 \delta c$, and $\varepsilon= \pm 1$, we obtain

$$
\begin{aligned}
& u_{11}(x, t) \\
& =\frac{3 c}{\mu}\left(1+\varepsilon \tanh \left(\frac{\sqrt{c}}{2}\left(\frac{x^{\alpha}}{\Gamma(1+\alpha)}-\frac{c t^{\alpha}}{\Gamma(1+\alpha)}\right)\right)\right), \\
& u_{12}(x, t) \\
& =\frac{3 c}{\mu}\left(1+\varepsilon \operatorname{coth}\left(\frac{\sqrt{c}}{2}\left(\frac{x^{\alpha}}{\Gamma(1+\alpha)}-\frac{c t^{\alpha}}{\Gamma(1+\alpha)}\right)\right)\right) .
\end{aligned}
$$

The evolution of exact solution for (30)-(41) is described in Figures 7-13.

It can be observed from (30)-(41) that we have successfully obtained twelve exact analytical solutions of the space-time fractional combined $\mathrm{KdV}-\mathrm{mKdV}$ equation. In comparison, [37] using the fractional mapping method only obtained five analytical solutions; thus, the proposed lookup table method is more concise and more effective.

3.3. The $(2+1)$-Dimensional Time Fractional Zoomeron Equation. Now, we use the proposed methods to construct the exact solutions of the following nonlinear $(2+1)$ dimensional time fractional Zoomeron equation [39]:

$$
D_{t t}^{2 \alpha}\left(\frac{u_{x y}}{u}\right)-\left(\frac{u_{x y}}{u}\right)_{x x}+D_{t}^{\alpha} 2\left(u^{2}\right)_{x}=0,
$$

$$
0<\alpha \leq 1 \text {, }
$$

where $u(x, y, t)$ is the amplitude of the relative mode.

We perform the fractional complex transformation:

$$
u(x, y, t)=u(\xi), \quad \xi=x+y-\frac{c t^{\alpha}}{\Gamma(1+\alpha)} .
$$

Substituting (43) into (42) and utilizing (2), we can reduce (42) into an ODE of integer order as follows:

$$
c^{2}\left(\frac{u^{\prime \prime}}{u}\right)^{\prime \prime}-\left(\frac{u^{\prime \prime}}{u}\right)^{\prime \prime}+2 c\left(u^{2}\right)^{\prime \prime}=0 .
$$

Integrating (44) twice with respect to $\xi$, we obtain

$$
\left(c^{2}-1\right) u^{\prime \prime}-2 c u^{3}+R u=0,
$$

where $R$ is the first integral constant $(R \neq 0)$ and the second integral constant is set to zero.

Multiplying (45) by $u^{\prime}$ and then integrating once again, we obtain

$$
\left(u^{\prime}\right)^{2}=a_{0}+a_{2} u^{2}+a_{4} u^{4}
$$

where $a_{2}=c /\left(c^{2}-1\right), a_{4}=-R /\left(c^{2}-1\right)$, and $a_{0}$ is an integral constant.

According to the characteristics of (46), looking up Table 1 and determining the corresponding coefficients and existence conditions of the exact solutions, we obtain several types of solutions to (42) as follows:

(1) When $c>1, R>0, a_{0}=0$, and $\varepsilon= \pm 1$, we obtain

$$
\begin{aligned}
& u_{1}(x, y, t) \\
& =\sqrt{\frac{c}{R}} \operatorname{sech}\left(\sqrt{\frac{c}{c^{2}-1}}\left(x+y-\frac{c t^{\alpha}}{\Gamma(1+\alpha)}\right)\right), \\
& \begin{aligned}
u_{2} & (x, y, t) \\
& =\sqrt{\frac{c}{R}} \operatorname{csch}\left(\sqrt{\frac{c}{c^{2}-1}}\left(x+y-\frac{c t^{\alpha}}{\Gamma(1+\alpha)}\right)\right) .
\end{aligned}
\end{aligned}
$$


TABLE 2: Solutions of (29), $\Delta=a_{3}^{2}-4 a_{2} a_{4}, \varepsilon= \pm 1$.

\begin{tabular}{|c|c|}
\hline Number & $\varphi$ \\
\hline 1 & $\frac{-a_{2} a_{3} \operatorname{sech}^{2}\left(\left(\sqrt{a_{2}} / 2\right) \xi\right)}{a_{3}-a_{2} a_{4}\left(1+\varepsilon \tanh \left(\left(\sqrt{a_{2}} / 2\right) \xi\right)\right)^{2}}, a_{2}>0$ \\
\hline 2 & $\frac{-a_{2} a_{3} \operatorname{csch}^{2}\left(\left(\sqrt{a_{2}} / 2\right) \xi\right)}{a_{3}-a_{2} a_{4}\left(1+\varepsilon \operatorname{coth}\left(\left(\sqrt{a_{2}} / 2\right) \xi\right)\right)^{2}}, a_{2}>0$ \\
\hline 3 & $\frac{2 a_{2} \operatorname{sech}\left(\sqrt{a_{2}} \xi\right)}{\varepsilon \sqrt{\Delta}-a_{3} \operatorname{sech}\left(\sqrt{a_{2}} \xi\right)}, a_{2}>0, \Delta>0$ \\
\hline 4 & $\frac{2 a_{2} \sec \left(\sqrt{-a_{2}} \xi\right)}{\varepsilon \sqrt{\Delta}-a_{3} \sec \left(\sqrt{-a_{2}} \xi\right)}, a_{2}<0, \Delta>0$ \\
\hline 5 & $\frac{2 a_{2} \csc \left(\sqrt{-a_{2}} \xi\right)}{\varepsilon \sqrt{-\Delta}-a_{3} \csc \left(\sqrt{-a_{2}} \xi\right)}, a_{2}<0, \Delta>0$ \\
\hline 6 & $\frac{2 a_{2} \operatorname{csch}\left(\sqrt{a_{2}} \xi\right)}{\varepsilon \sqrt{-\Delta}-a_{3} \operatorname{csch}\left(\sqrt{a_{2}} \xi\right)}, a_{2}>0, \Delta<0$ \\
\hline 7 & $\frac{-a_{2} \operatorname{sech}^{2}\left(\left(\sqrt{a_{2}} / 2\right) \xi\right)}{a_{3}+2 \varepsilon \sqrt{a_{2} a_{4}} \tanh \left(\left(\sqrt{a_{2}} / 2\right) \xi\right)}, a_{2}>0, a_{4}>0$ \\
\hline 8 & $\frac{a_{2} a_{3} \operatorname{csch}^{2}\left(\left(\sqrt{a_{2}} / 2\right) \xi\right)}{a_{3}^{2}-a_{2} a_{4}\left(1+\varepsilon \operatorname{coth}\left(\left(\sqrt{a_{2}} / 2\right) \xi\right)\right)}, a_{2}>0, a_{4}>0$ \\
\hline 9 & $\frac{-a_{2} \operatorname{sech}^{2}\left(\left(\sqrt{-a_{2}} / 2\right) \xi\right)}{a_{3}+2 \varepsilon \sqrt{-a_{2} a_{4}} \tan \left(\left(\sqrt{-a_{2}} / 2\right) \xi\right)}, a_{2}<0, a_{4}>0$ \\
\hline 10 & $\frac{-a_{2} \csc ^{2}\left(\left(\sqrt{-a_{2}} / 2\right) \xi\right)}{a_{3}+2 \varepsilon \sqrt{-a_{2} a_{4}} \cot \left(\left(\sqrt{-a_{2}} / 2\right) \xi\right)}, a_{2}<0, a_{4}>0$ \\
\hline 11 & $-\frac{a_{2}}{a_{3}}\left(1+\varepsilon \tanh \left(\frac{\sqrt{a_{2}}}{2} \xi\right)\right), a_{2}>0, \Delta=0$ \\
\hline 12 & $-\frac{a_{2}}{a_{3}}\left(1+\varepsilon \operatorname{coth}\left(\frac{\sqrt{a_{2}}}{2} \xi\right)\right), a_{2}>0, \Delta=0$ \\
\hline
\end{tabular}

(2) When $c<-1, R<0, a_{0}=0$, and $\varepsilon= \pm 1$, we obtain

$$
\begin{aligned}
& u_{3}(x, y, t) \\
& =\sqrt{\frac{c}{R}} \sec \left(\sqrt{-\frac{c}{c^{2}-1}}\left(x+y-\frac{c t^{\alpha}}{\Gamma(1+\alpha)}\right)\right), \\
& u_{4}(x, y, t) \\
& =\sqrt{\frac{c}{R}} \csc \left(\sqrt{-\frac{c}{c^{2}-1}}\left(x+y-\frac{c t^{\alpha}}{\Gamma(1+\alpha)}\right)\right) .
\end{aligned}
$$

(3) When $c<-1, R<0, a_{0}=-c^{2} / 4 R\left(c^{2}-1\right)$, and $\varepsilon= \pm 1$, we obtain

$$
\begin{array}{r}
u_{5}(x, y, t)=\varepsilon \sqrt{\frac{c}{2 R}} \\
\cdot \tanh \left(\sqrt{-\frac{c}{2\left(c^{2}-1\right)}}\left(x+y-\frac{c t^{\alpha}}{\Gamma(1+\alpha)}\right)\right), \\
u_{6}(x, y, t)=\varepsilon \sqrt{\frac{c}{2 R}}\left(\sqrt{-\frac{c}{2\left(c^{2}-1\right)}}\left(x+y-\frac{c t^{\alpha}}{\Gamma(1+\alpha)}\right)\right) .
\end{array}
$$

(4) When $c>1, R<0, a_{0}=-c^{2} / 4 R\left(c^{2}-1\right)$, and $\varepsilon= \pm 1$, we obtain

$$
\begin{aligned}
& u_{7}(x, y, t) \\
& =\varepsilon \sqrt{-\frac{c}{R}} \tan \left(\sqrt{\frac{c}{2\left(c^{2}-1\right)}}\left(x+y-\frac{c t^{\alpha}}{\Gamma(1+\alpha)}\right)\right), \\
& u_{8}(x, y, t) \\
& =\varepsilon \sqrt{-\frac{c}{R}} \cot \left(\sqrt{\frac{c}{2\left(c^{2}-1\right)}}\left(x+y-\frac{c t^{\alpha}}{\Gamma(1+\alpha)}\right)\right) .
\end{aligned}
$$

(5) When $c>1, R>0, a_{0}=\left(1-m^{2}\right) c /\left(2 m^{2}-1\right)^{2}\left(c^{2}-1\right)$, and $\varepsilon= \pm 1$, we obtain

$$
\begin{aligned}
& u_{9}(x, y, t)=\sqrt{\frac{c m^{2}}{R\left(2 m^{2}-1\right)}} \\
& \cdot \operatorname{cn}\left(\sqrt{\frac{c}{\left(c^{2}-1\right)\left(2 m^{2}-1\right)}}\left(x+y-\frac{c t^{\alpha}}{\Gamma(1+\alpha)}\right)\right) .
\end{aligned}
$$

(6) When $c<-1, R<0, a_{0}=-c^{2} m^{2} / 2 R\left(c^{2}-1\right)\left(m^{2}+1\right)$, and $\varepsilon= \pm 1$, we obtain

$$
\begin{aligned}
& u_{10}(x, y, t)=\varepsilon \sqrt{\frac{c m^{2}}{R\left(m^{2}+1\right)}} \\
& \cdot \operatorname{sn}\left(\sqrt{-\frac{c}{\left(c^{2}-1\right)\left(m^{2}+1\right)}}\left(x+y-\frac{c t^{\alpha}}{\Gamma(1+\alpha)}\right)\right) .
\end{aligned}
$$

By the way, when taking $y=0$, (42) and (9) have the same type solutions, so that we no longer give figures of the obtained solutions of (42) in this section.

If we take $k=\varepsilon=1, \sigma=c /\left(2\left(c^{2}-1\right)\right), A=c$, and $R=-c$, then the expression of $u_{5}(x, y, t)$ is the same as that of $u_{1}(x, y, t)$ for Eq. (27) in [39], the expression of $u_{6}(x, y, t)$ is the same as that of $u_{2}(x, y, t)$ for Eq. (27) in [39], and they can be, respectively, expressed as follows:

$$
\begin{aligned}
& u(x, y, t) \\
& =\sqrt{\frac{1}{2}} \tanh \left(\sqrt{-\frac{c}{2\left(c^{2}-1\right)}}\left(x+y-\frac{c t^{\alpha}}{\Gamma(1+\alpha)}\right)\right), \\
& u(x, y, t) \\
& \quad=\sqrt{\frac{1}{2}} \operatorname{coth}\left(\sqrt{-\frac{c}{2\left(c^{2}-1\right)}}\left(x+y-\frac{c t^{\alpha}}{\Gamma(1+\alpha)}\right)\right) .
\end{aligned}
$$

If we take $k=\varepsilon=1, \sigma=c /\left(2\left(c^{2}-1\right)\right), A=c$, and $R=-2 c$, then the expression of $u_{7}(x, y, t)$ is the same as that of $u_{3}(x, y, t)$ for Eq. (27) in [39], the expression of $u_{8}(x, y, t)$ 

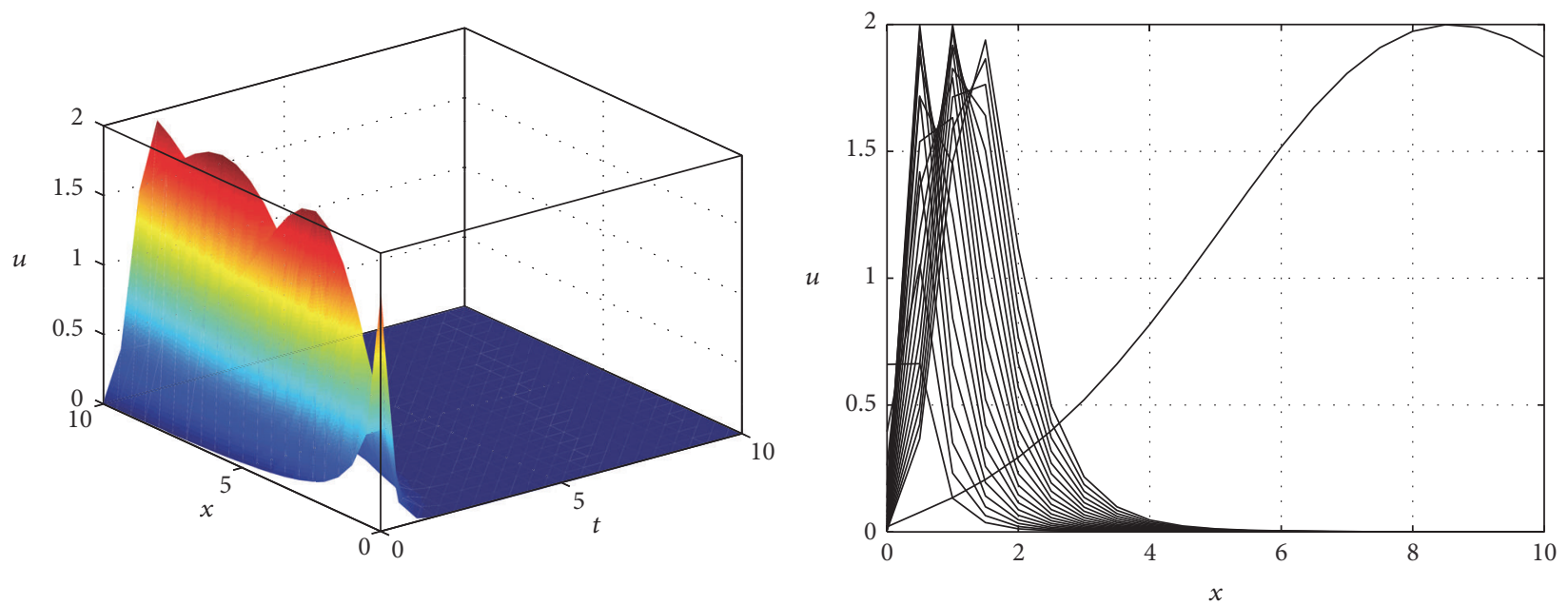

Figure 7: Exact solutions $u_{1}(x, t)$ for (30) and its position at $t=1.2$, when the parameters $\mu=2, \delta=1, c=3$, and $\alpha=0.5$.
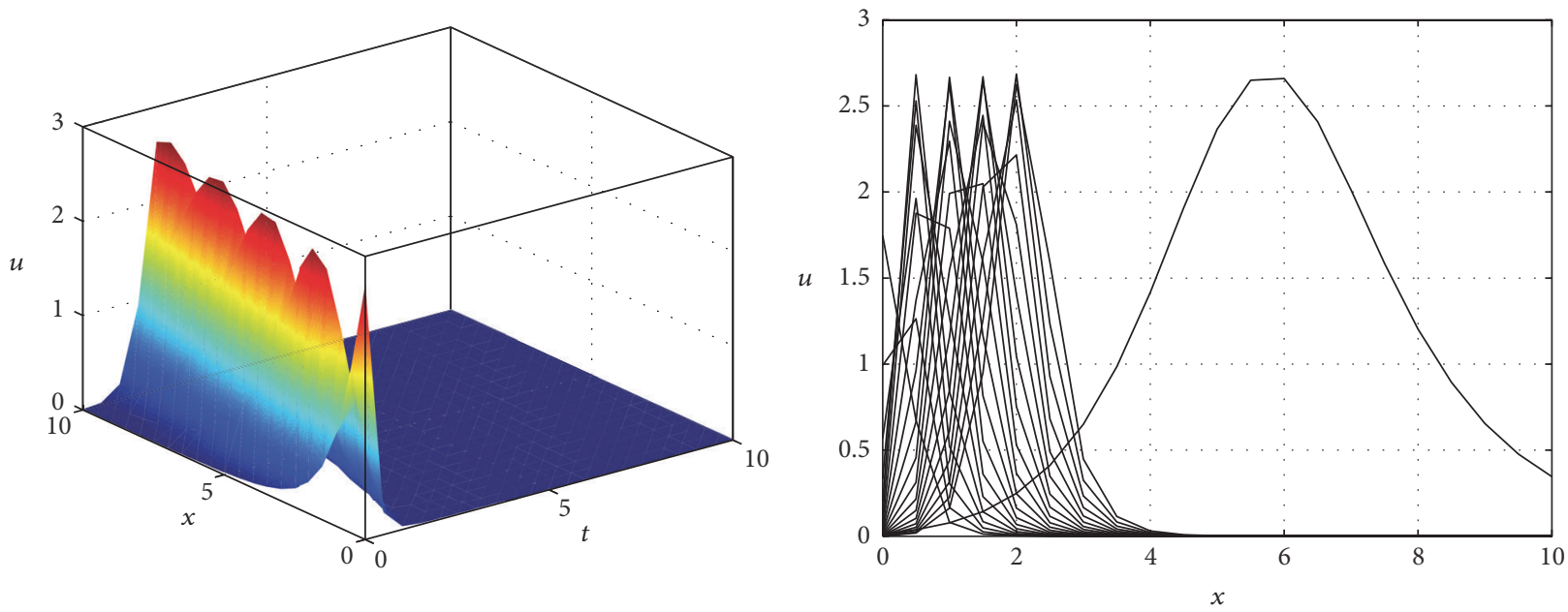

FIGURE 8: Exact solutions $u_{3}(x, t)$ for (32) and its position at $t=1.2$, when the parameters $\mu=2, \delta=1, c=3, \varepsilon=1$, and $\alpha=0.7$.
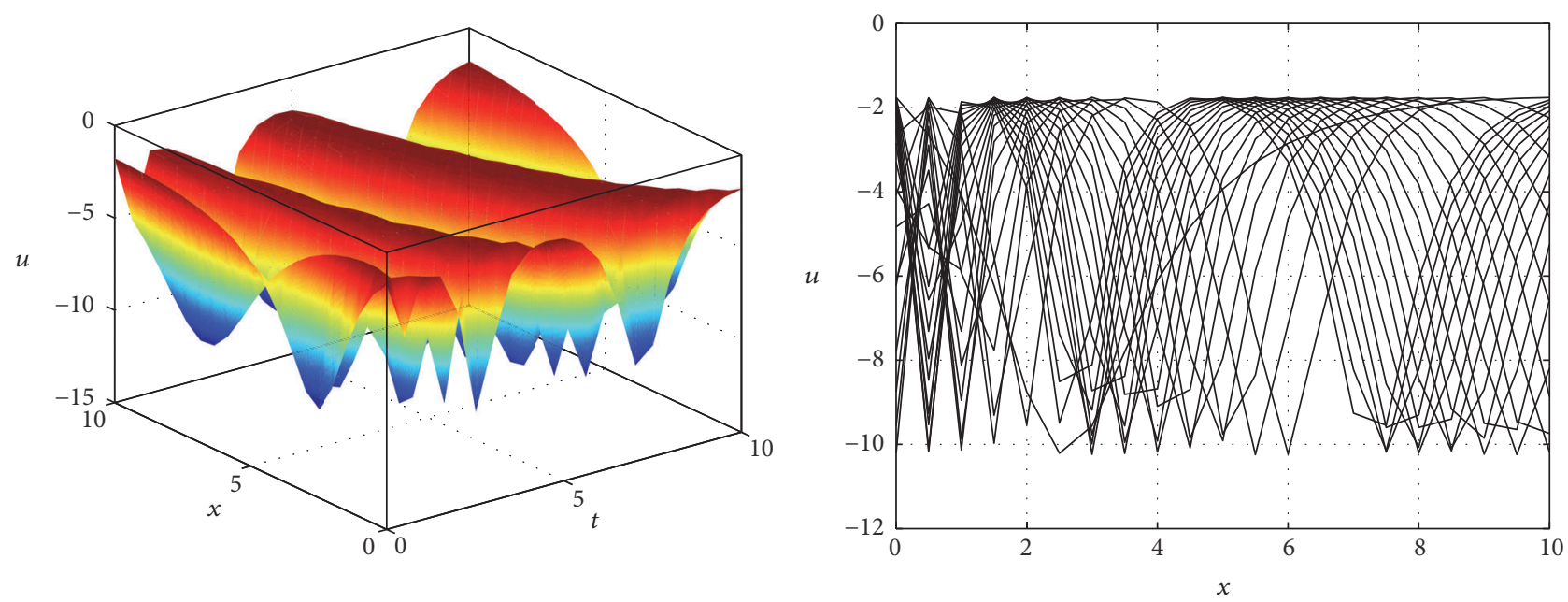

FIgURE 9: Exact solutions $u_{4}(x, t)$ for (33) and its position at $t=0$, when the parameters $\mu=6, \delta=1, c=-3, \varepsilon=1$, and $\alpha=0.5$. 

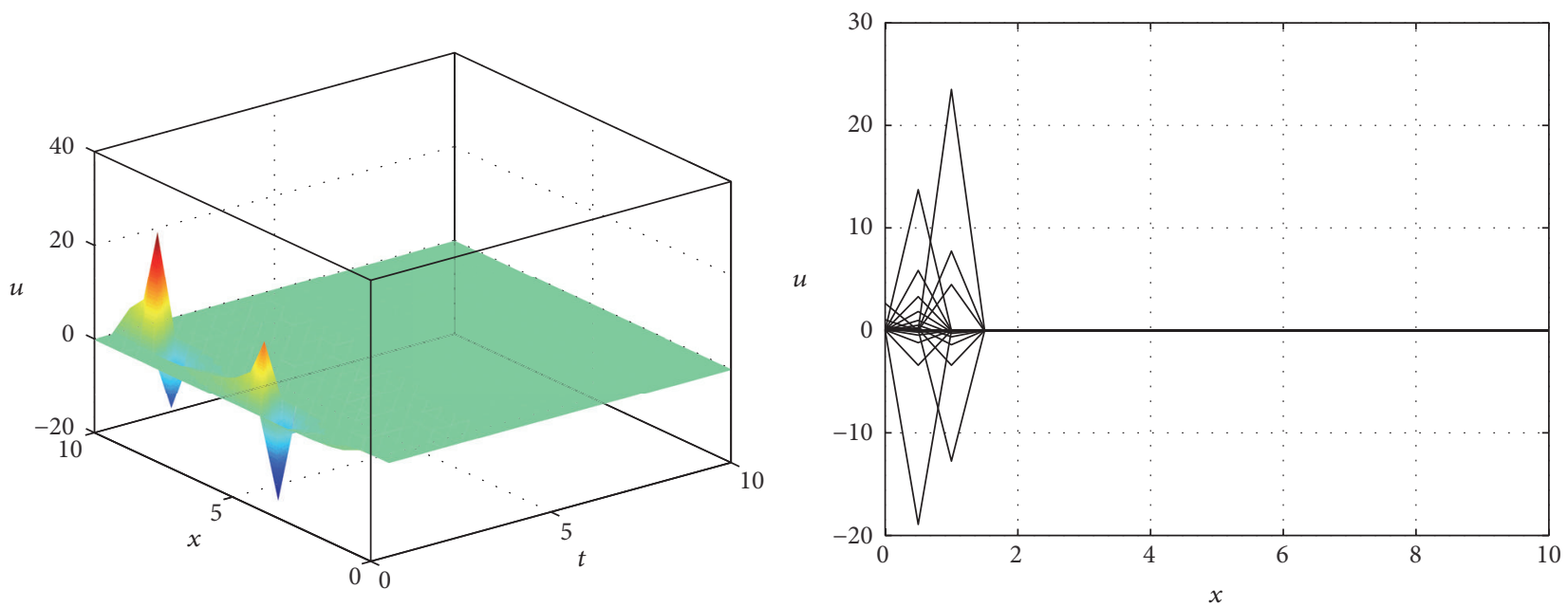

FIGURE 10: Exact solutions $u_{6}(x, t)$ for (35) and its position at $t=0$, when the parameters $\mu=4, \delta=-1, c=6, \varepsilon=1$, and $\alpha=0.8$.
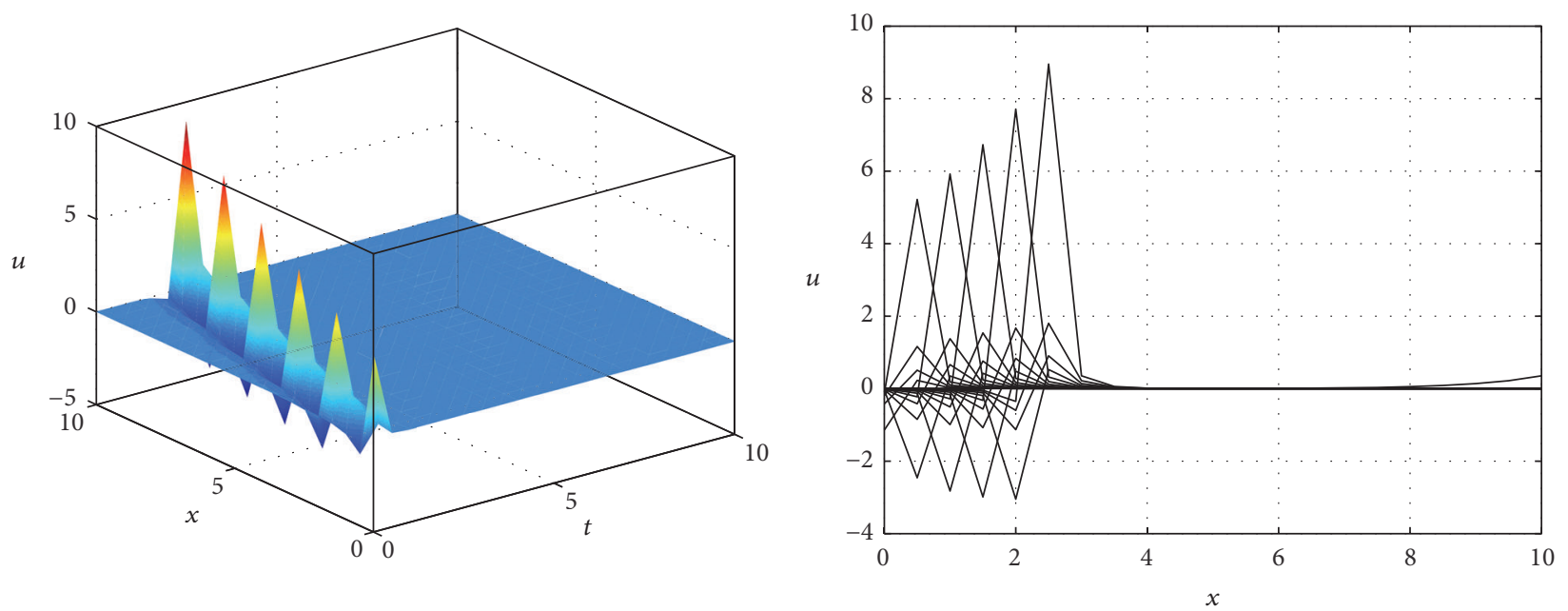

Figure 11: Exact solutions $u_{7}(x, t)$ for (36) and its position at $t=3$, when the parameters $\mu=2, \delta=-15, c=3, \varepsilon=1$, and $\alpha=0.8$.
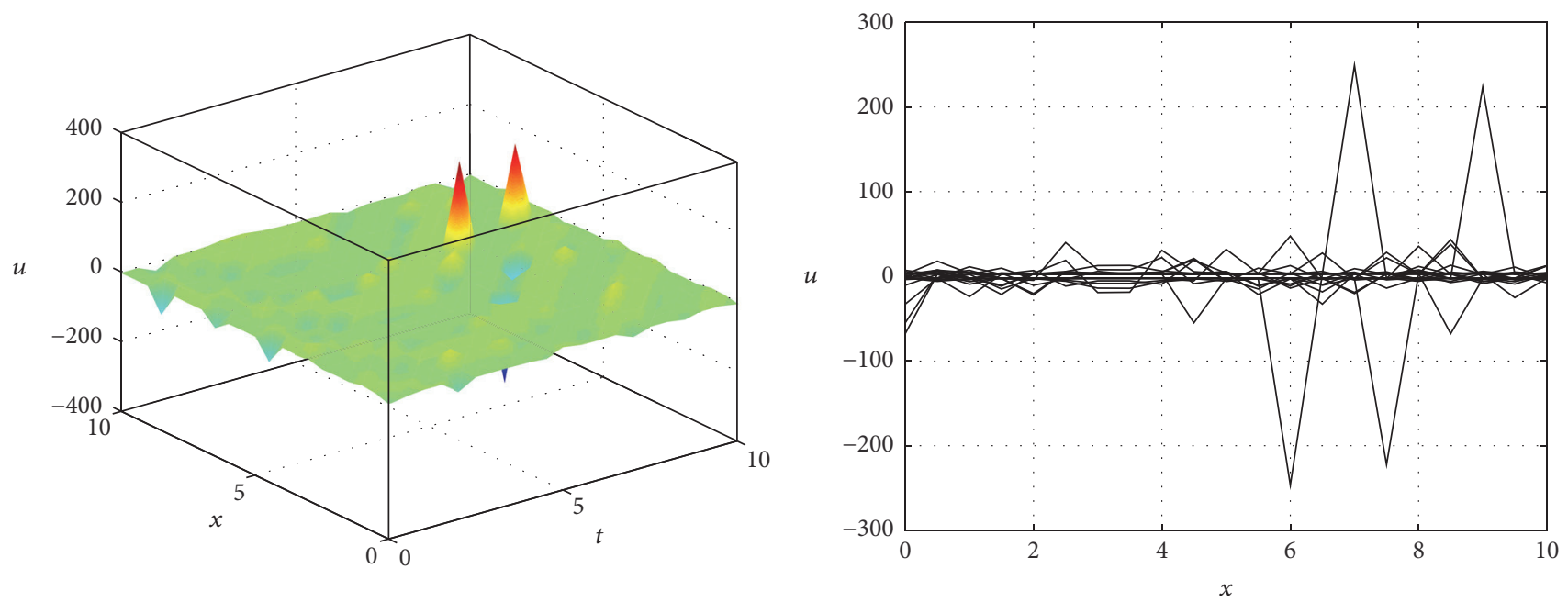

Figure 12: Exact solutions $u_{9}(x, t)$ for (38) and its position at $t=0$, when the parameters $\mu=2, \delta=-10, c=-7, \varepsilon=1$, and $\alpha=0.8$. 

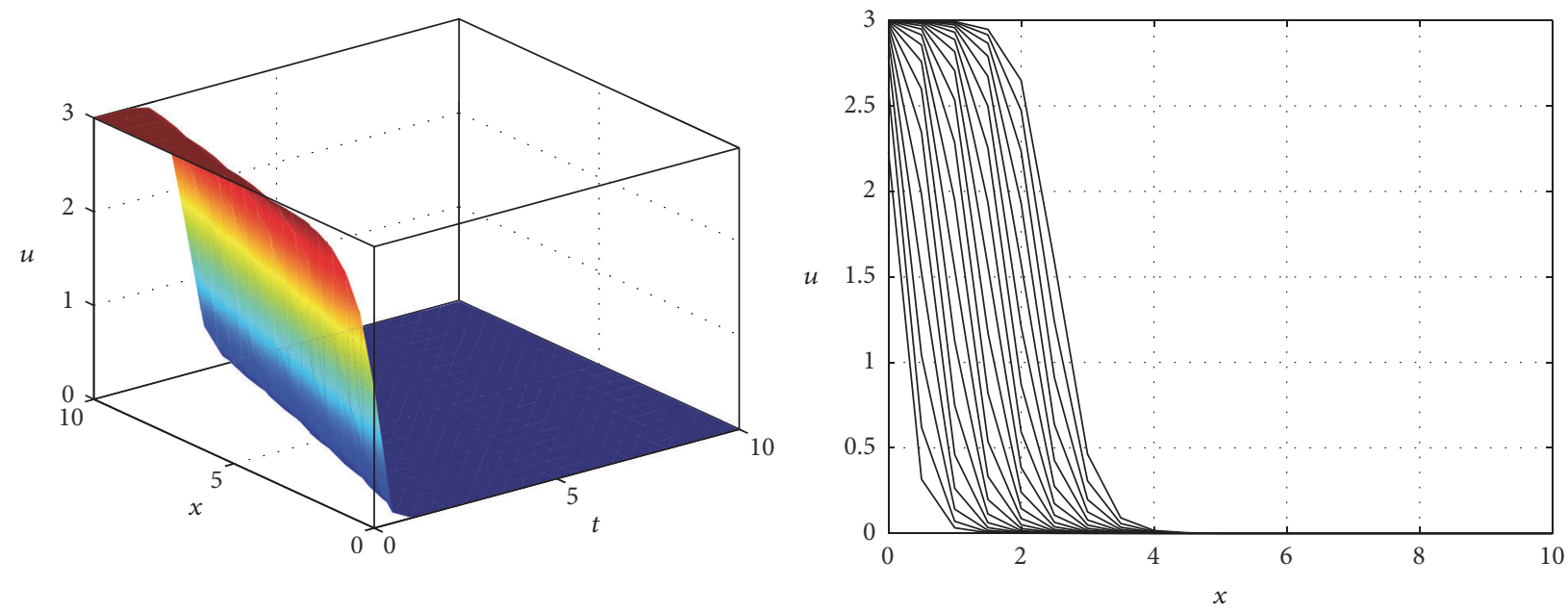

Figure 13: Exact solutions $u_{11}(x, t)$ for $(40)$ and its position at $t=0$, when the parameters $\mu=6, \delta=-2, c=3, \varepsilon=1$, and $\alpha=0.8$.

is the same as that of $u_{4}(x, y, t)$ for Eq. (27) in [39], and they can be, respectively, expressed as follows:

$$
\begin{aligned}
& u(x, y, t) \\
& =-\sqrt{\frac{1}{2}} \tan \left(\sqrt{-\frac{c}{2\left(c^{2}-1\right)}}\left(x+y-\frac{c t^{\alpha}}{\Gamma(1+\alpha)}\right)\right), \\
& u(x, y, t) \\
& =\sqrt{\frac{1}{2}} \cot \left(\sqrt{-\frac{c}{2\left(c^{2}-1\right)}}\left(x+y-\frac{c t^{\alpha}}{\Gamma(1+\alpha)}\right)\right) .
\end{aligned}
$$

However, we also obtain the bell-shaped solitary wave solution and Jacobi elliptic function solution of the $(2+1)$ dimension time fractional Zoomeron equation.

3.4. The Space-Time Fractional ZKBBM Equation. Next, we solve the exact solution of the following space-time fractional nonlinear Zakharov-Kuznetsov-Benjamin-BonaMahony (ZKBBM) equation [40]:

$$
\begin{aligned}
\frac{\partial^{\alpha} u}{\partial t^{\alpha}}+\frac{\partial^{\beta} u}{\partial x^{\beta}}-2 a u \frac{\partial^{\beta} u}{\partial x^{\beta}}-b \frac{\partial^{\alpha}}{\partial t^{\alpha}}\left(\frac{\partial^{2 \beta} u}{\partial x^{2 \beta}}\right) & =0, \\
t>0, \alpha & >0, \beta \leq 1,
\end{aligned}
$$

where $a$ and $b$ are arbitrary nonzero constants.

We first use the fractional complex transform $u(x, t)=$ $u(\xi), \xi=x^{\beta} / \Gamma(1+\beta)-c t^{\alpha} / \Gamma(1+\alpha)$, and (55) becomes an ODE of integer order:

$$
(1-c) u^{\prime}-2 a u u^{\prime}+b c u^{\prime \prime \prime}=0 .
$$
yields

Integrating (56) once with an integral constant of zero

$$
(1-c) u-a u^{2}+b c u^{\prime \prime}=0 .
$$

TABLE 3: Solutions of (59).

\begin{tabular}{ll}
\hline Number & $\varphi$ \\
\hline 1 & $\varphi=-\frac{a_{2}}{a_{3}} \operatorname{sech}^{2}\left(\frac{\sqrt{a_{2}}}{2} \xi\right), a_{2}>0, a_{0}=0, a_{1}=0$ \\
2 & $\varphi=-\frac{a_{2}}{a_{3}} \sec ^{2}\left(\frac{\sqrt{-a_{2}}}{2} \xi\right), a_{2}<0, a_{0}=0, a_{1}=0$ \\
3 & $\varphi=\frac{1}{a_{3} \xi^{2}}, a_{2}=0, a_{0}=0, a_{1}=0$ \\
\hline
\end{tabular}

Multiplying (57) by $u^{\prime}$ and integrating once again with an integral constant of zero, we obtain

$$
\left(u^{\prime}\right)^{2}=a_{2} u^{2}+a_{3} u^{3},
$$

where $a_{2}=(c-1) / b c$ and $a_{3}=2 a / 3 b c$.

According to the solutions of the auxiliary equation presented in [35], the exact solutions of the (59) are listed in Table 3:

$$
\left(\varphi^{\prime}\right)^{2}=a_{2} \varphi^{2}+a_{3} \varphi^{3} .
$$

Equations (58) and (59) have the same form. Thus, looking up Table 3 and determining the coefficients and existence conditions, we can obtain the following solutions of (55):

(1) If $c>1$ and $b>0$, we obtain

$$
\begin{aligned}
& u_{1}(x, t)=-\frac{3(c-1)}{2 a} \\
& \cdot \operatorname{sech}^{2}\left(\frac{1}{2} \sqrt{\frac{c-1}{b c}}\left(\frac{x^{\beta}}{\Gamma(1+\beta)}-\frac{c t^{\alpha}}{\Gamma(1+\alpha)}\right)\right) .
\end{aligned}
$$



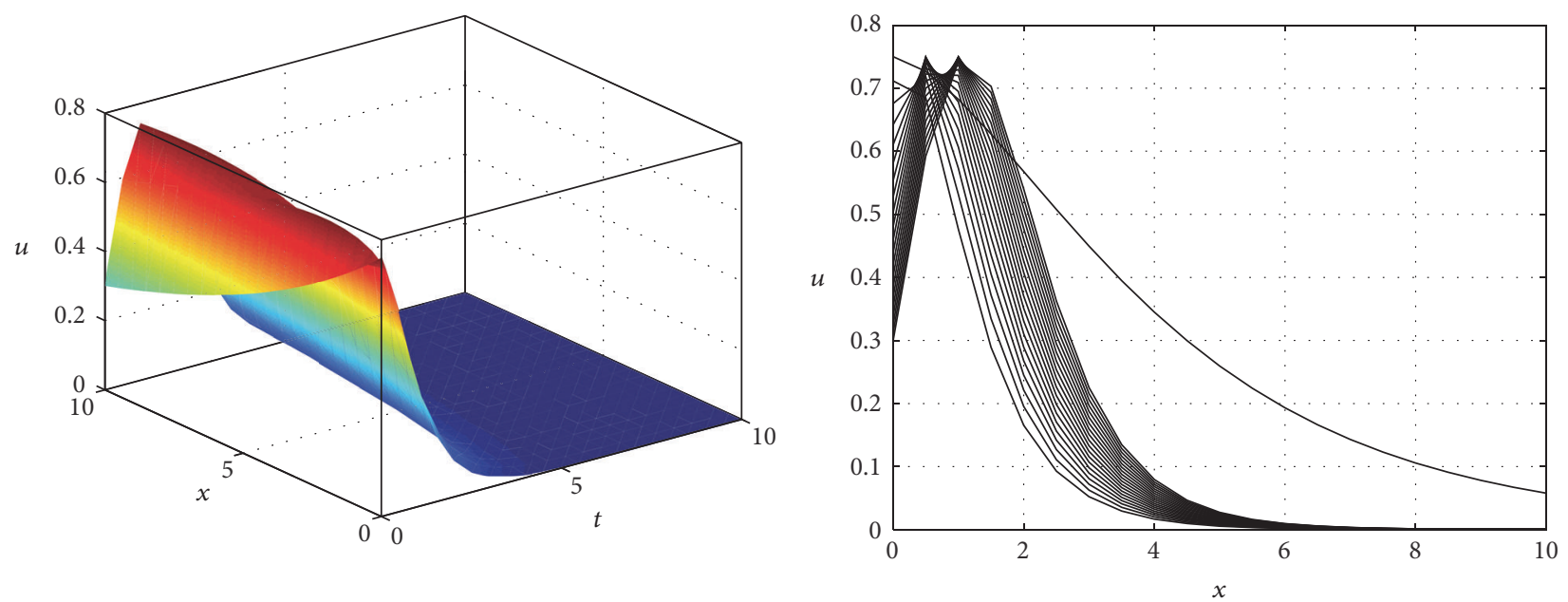

FIGURE 14: Exact solutions $u_{1}(x, t)$ for (60) and its position at $t=0$, when the parameters $a=-4, b=2, c=3, \alpha=0.8$, and $\beta=0.5$.
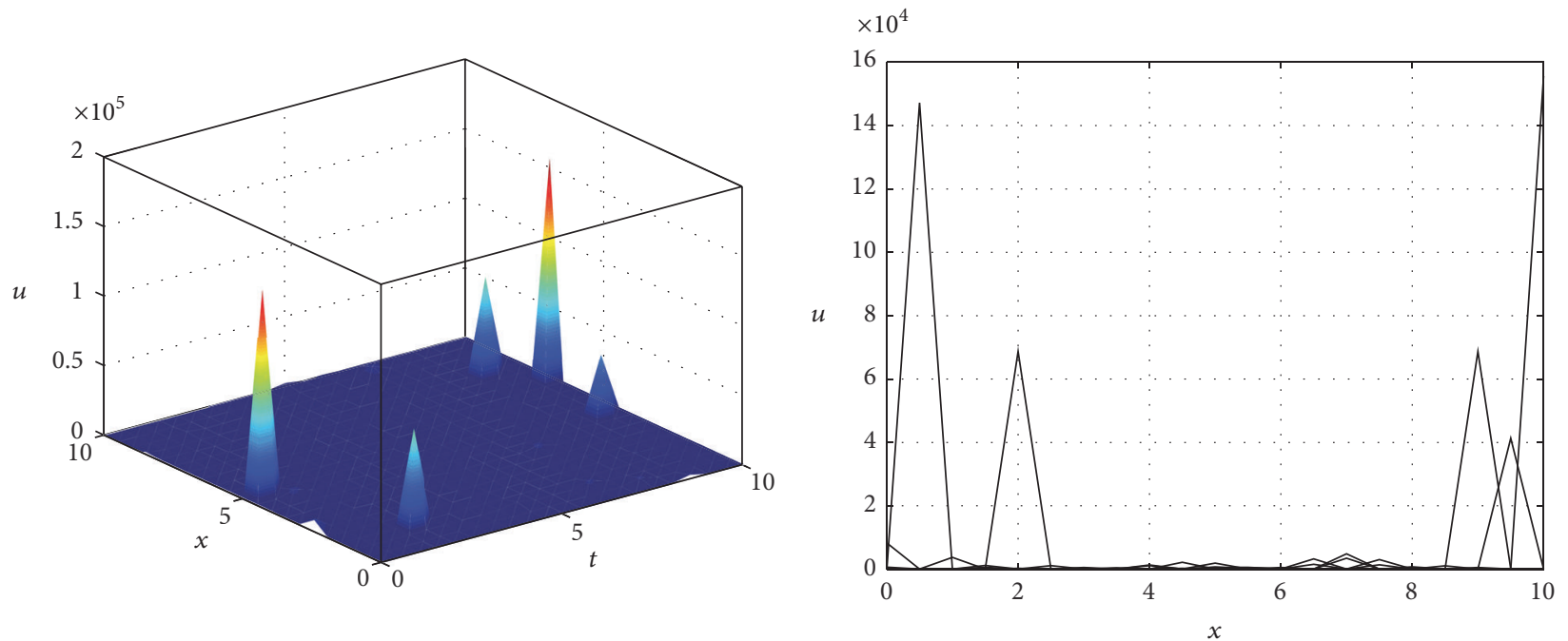

FIGURE 15: Exact solutions $u_{2}(x, t)$ for $(61)$ and its position at $t=5$, when the parameters $a=-4, b=-0.5, c=10, \alpha=0.7$, and $\beta=0.9$.

(2) If $c>1$ and $b<0$, we obtain

$$
\begin{aligned}
& u_{2}(x, t)=-\frac{3(c-1)}{2 a} \\
& \cdot \sec ^{2}\left(\frac{1}{2} \sqrt{-\frac{c-1}{b c}}\left(\frac{x^{\beta}}{\Gamma(1+\beta)}-\frac{c t^{\alpha}}{\Gamma(1+\alpha)}\right)\right) .
\end{aligned}
$$

(3) If $c=1$, we obtain

$$
u_{3}(x, t)=\frac{2 a}{3 b c\left(x^{\beta} / \Gamma(1+\beta)-c t^{\alpha} / \Gamma(1+\alpha)\right)^{2}} .
$$

The asymptotic behavior for the obtained exact solution (60)-(62) is shown in Figures 14-16.

It can be observed from Figures 14-16 that we obtained single solitary wave and multiple solitary wave solutions of fractional ZKBBM equation, and when $\alpha=\beta$, (62) is singular wave solution, and when $\alpha \neq \beta$, (62) is multiple solitary wave solution. Consequently, the exact solution of fractional differential equation can better describe the complex wave propagation. Reference [40] only obtained Jacobi elliptic doubly periodic solution of fractional ZKBBM equation, but we obtain three types of solutions using our proposed method.

\section{Conclusions}

In this paper, by looking up the tables of solutions of corresponding auxiliary equations, the exact analytical solutions of the time fractional simplified $\mathrm{MCH}$ equation, the space-time fractional combined $\mathrm{KdV}-\mathrm{mKdV}$ equation, the $(2+1)$-dimensional time fractional Zoomeron equation, and the space-time fractional ZKBBM equation have been constructed directly and successfully. Compared with previous results, solutions of more types are obtained, some of 


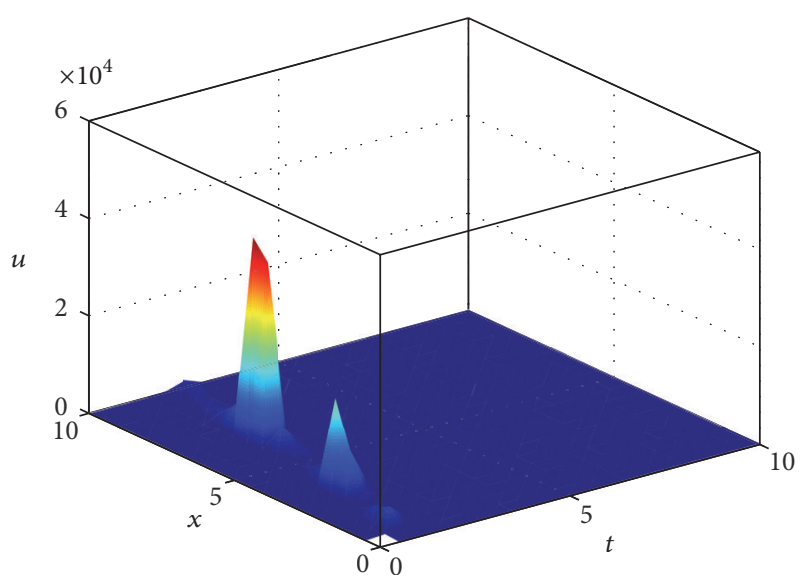

(a)

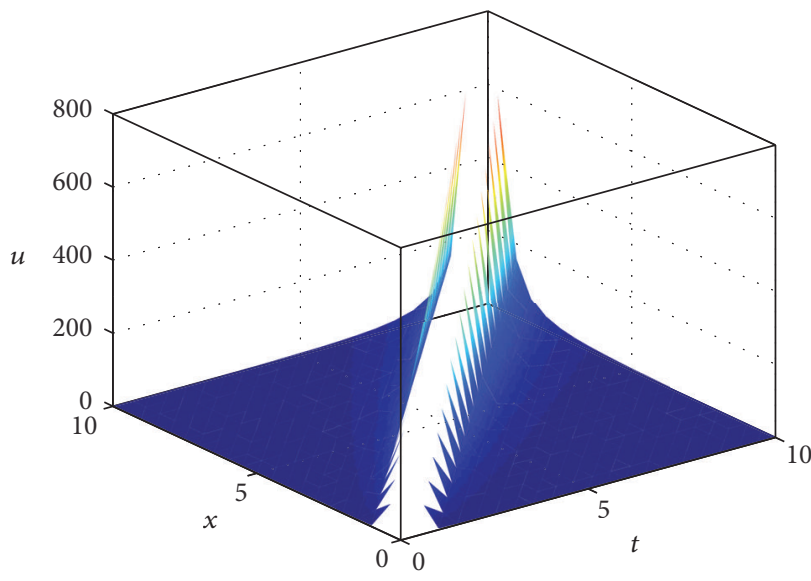

(b)

Figure 16: Exact solutions $u_{3}(x, t)$ for (62): (a) $\alpha=0.5, \beta=0.2$; (b) $\alpha=\beta=0.5$, when the parameters $a=-4, b=-0.5$, and $c=10$.

which are new. Furthermore, the solving process is more concise and straightforward. Consequently, the table lookup method is concluded to be effective method and would be a powerful mathematical tool for obtaining more exact analytical solutions of a large number of nonlinear fPDEs.

\section{Competing Interests}

The authors declare that there is no conflict of interests regarding the publication of this article and regarding the funding that they have received.

\section{Acknowledgments}

The work was supported by the National Natural Science Foundation of China (61673222, 51607004), the Major Project of Nature Science Foundation of Higher Education Institution of Jiangsu Province, China (13KJA510001), the Project of Department of Education of Anhui Province, China (AQKJ2015B015), Research Innovation Program for College Graduates of Jiangsu Province, China (KYLX15 _0873), Anhui Provincial Natural Science Foundation, China (1608085QF157), and Key Projects of Anhui Province University Outstanding Youth Talent Support Program, China (gxyqZD2016207).

\section{References}

[1] X. Wen, L. Shao, Y. Xue, and W. Fang, "A rapid learning algorithm for vehicle classification," Information Sciences, vol. 295, no. 1, pp. 395-406, 2015.

[2] Z. Xia, X. Wang, X. Sun, Q. Liu, and N. Xiong, "Steganalysis of LSB matching using differences between nonadjacent pixels," Multimedia Tools and Applications, vol. 75, no. 4, pp. 1947-1962, 2016.

[3] O. H. Mohammed and A. I. Khlaif, "Adomian decomposition method for solving delay differential equations of fractional order," IOSR Journal of Mathematics, vol. 10, no. 6, pp. 1-5, 2014.
[4] M. A. E. Herzallah and K. A. Gepreel, "Approximate solution to the time-space fractional cubic nonlinear Schrodinger equation," Applied Mathematical Modelling, vol. 36, no. 11, pp. 5678$5685,2012$.

[5] J. S. Duan, T. Chaolu, R. Rach, and L. Lu, "The Adomian decomposition method with convergence acceleration techniques for nonlinear fractional differential equations," Computers \& Mathematics with Applications, vol. 66, no. 5, pp. 728-736, 2013.

[6] A. M. A. El-Sayed, S. H. Behiry, and W. E. Raslan, "Adomian's decomposition method for solving an intermediate fractional advection-dispersion equation," Computers \& Mathematics with Applications, vol. 59, no. 5, pp. 1759-1765, 2010.

[7] G.-C. Wu and D. Baleanu, "Variational iteration method for the Burgers' flow with fractional derivatives-new Lagrange multipliers," Applied Mathematical Modelling, vol. 37, no. 9, pp. 6183-6190, 2013.

[8] G. Wu and E. W. Lee, "Fractional variational iteration method and its application," Physics Letters. A, vol. 374, no. 25, pp. 25062509, 2010.

[9] S. Guo and L. Mei, "The fractional variational iteration method using He's polynomials," Physics Letters. A, vol. 375, no. 3, pp. 309-313, 2011.

[10] A. Golbabai and K. Sayevand, "Fractional calculus-a new approach to the analysis of generalized fourth-order diffusion wave equations," Computers \& Mathematics with Applications, vol. 61, no. 8, pp. 2227-2231, 2011.

[11] K. A. Gepreel, “The homotopy perturbation method applied to the nonlinear fractional Kolmogorov-Petrovskii-Piskunov equations," Applied Mathematics Letters, vol. 24, no. 8, pp. 1428$1434,2011$.

[12] T. A. Nofel, "Application of the homotopy perturbation method to nonlinear heat conduction and fractional van der pol damped nonlinear oscillator," Applied Mathematics, vol. 5, no. 6, pp. 852861, 2014.

[13] S. Das and P. K. Gupta, "An approximate analytical solution of the fractional diffusion equation with absorbent term and external force by homotopy perturbation method," Zeitschrift Für Naturforschung A, vol. 65, no. 3, pp. 182-190, 2014.

[14] K. A. Gepreel and M. S. Mohamed, "Analytical approximate solution for nonlinear space-time fractional Klein-Gordon 
equation," Chinese Physics B, vol. 22, no. 1, Article ID 010201, 2013.

[15] M. S. Mohamed, F. Al-Malki, and R. Talib, "Jacobi elliptic numerical solutions for the time fractional dispersive long wave equation," International Journal of Pure and Applied Mathematics, vol. 80, no. 5, pp. 635-646, 2012.

[16] M. K. Bansal and R. Jain, "Application of generalized differential transform method to fractional order riccati differential equation and numerical result," International Journal of Pure and Applied Mathematics, vol. 99, no. 3, pp. 355-366, 2015.

[17] Q. Xu and J. S. Hesthaven, "Stable multi-domain spectral penalty methods for fractional partial differential equations," Journal of Computational Physics, vol. 257, pp. 241-258, 2014.

[18] M. Zayernouri and G. E. Karniadakis, "Exponentially accurate spectral and spectral element methods for fractional ODEs," Journal of Computational Physics, vol. 257, pp. 460-480, 2014.

[19] Q. Xu and J. S. Hesthaven, "Discontinuous Galerkin method for fractional convection-diffusion equations," SIAM Journal on Numerical Analysis, vol. 52, no. 1, pp. 405-423, 2014.

[20] G. Pang, W. Chen, and Z. Fu, "Space-fractional advectiondispersion equations by the Kansa method," Journal of Computational Physics, vol. 293, pp. 280-296, 2015.

[21] S. Zhang and H.-Q. Zhang, "Fractional sub-equation method and its applications to nonlinear fractional PDEs," Physics Letters A, vol. 375, no. 7, pp. 1069-1073, 2011.

[22] S. Guo, L. Mei, Y. Li, and Y. Sun, "The improved fractional sub-equation method and its applications to the space-time fractional differential equations in fluid mechanics," Physics Letters A, vol. 376, no. 4, pp. 407-411, 2012.

[23] B. Abdel-Salam, E. A. Yousif, and G. F. Hassan, "Solution of nonlinear space time fractional differential equations via the fractional projective Riccati expansion method," Physics, vol. 2013, no. 4, pp. 657-675, 2015.

[24] S. Zhang, J. Wang, A.-X. Peng, and B. Cai, "A generalized exp-function method for multiwave solutions of sine-Gordon equation," Pramana, vol. 81, no. 5, pp. 763-773, 2013.

[25] A. Bekir, Ö. Güner, and A. C. Cevikel, "Fractional complex transform and exp-function methods for fractional differential equations," Abstract and Applied Analysis, vol. 2013, Article ID 426462, 8 pages, 2013.

[26] B. Zheng, " $\left(G^{\prime} / G\right)$ expansion method for solving fractional partial differential equations in the theory of mathematical physics," Phys, vol. 58, no. 5, pp. 623-630, 2012.

[27] K. A. Gepreel and S. Omran, "Exact solutions for nonlinear partial fractional differential equations," Chinese Physics B, vol. 21, no. 11, Article ID 110204, 2012.

[28] A. Bekir, Ö. Güner, A. H. Bhrawy, and A. Biswas, "Solving nonlinear fractional differential equations using exp-function and (G'/G) -expansion methods," Romanian Journal of Physics, vol. 60, no. 3-4, pp. 360-378, 2015.

[29] W. Liu and K. Chen, "The functional variable method for finding exact solutions of some nonlinear time-fractional differential equations," Pramana, vol. 81, no. 3, pp. 377-384, 2013.

[30] M. Matinfar, M. Eslami, and M. Kordy, “The functional variable method for solving the fractional Korteweg-de Vries equations and the coupled Korteweg-de Vries equations," Pramana, vol. 85, no. 4, pp. 583-592, 2015.

[31] B. Lu, "The first integral method for some time fractional differential equations," Journal of Mathematical Analysis and Applications, vol. 395, no. 2, pp. 684-693, 2012.
[32] A. Bekir, O. Guner, and O. Unsal, "The first integral method for exact solutions of nonlinear fractional differential equations," Journal of Computational \& Nonlinear Dynamics, vol. 10, no. 2, Article ID 021020, 5 pages, 2015.

[33] G. Jumarie, "Modified Riemann-Liouville derivative and fractional Taylor series of nondifferentiable functions further results," Computers and Mathematics with Applications, vol. 51, no. 9-10, pp. 1367-1376, 2006.

[34] G. Jumarie, "Laplace's transform of fractional order via the Mittag-Leffler function and modified Riemann-Liouville derivative," Applied Mathematics Letters, vol. 22, no. 11, pp. 16591664, 2009.

[35] E. Fan, "A new algebraic method for finding the line soliton solutions and doubly periodic wave solution to a two-dimensional perturbed KdV equation," Chaos, Solitons \& Fractals, vol. 15, no. 3, pp. 567-574, 2003.

[36] M. Nur Alam and M. Ali Akbar, "Some new exact traveling wave solutions to the simplified $\mathrm{MCH}$ equation and the $(1+1)$ dimensional combined KdV-mKdV equations," Journal of the Association of Arab Universities for Basic \& Applied Sciences, vol. 17, pp. 6-13, 2015.

[37] A. B. Abdel-Salam and Z. I. A. Al-Muhiameed, "Analytic solutions of the space-time fractional combined KdV-mKdV equation," Mathematical Problems in Engineering, vol. 2015, Article ID 871635, 6 pages, 2015.

[38] B.-G. Zhang, Z.-R. Liu, and J.-F. Mao, "New exact solutions for $\mathrm{mCH}$ and $\mathrm{mDP}$ equations by auxiliary equation method," Applied Mathematics and Computation, vol. 217, no. 4, pp. 13061314, 2010.

[39] E. Aksoy, A. C. Çevikel, and A. Bekir, "Soliton solutions of (2+1)dimensional time-fractional Zoomeron equation," Optik, vol. 127, no. 17, pp. 6933-6942, 2016.

[40] K. A. Gepreel, "Explicit Jacobi elliptic exact solutions for nonlinear partial fractional differential equations," Advances in Difference Equations, vol. 2014, article 286, 2014. 


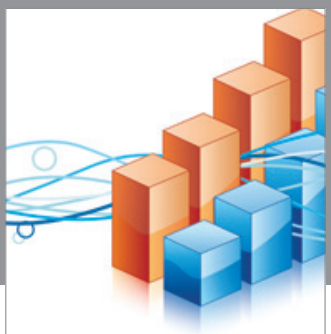

Advances in

Operations Research

vatem alat4

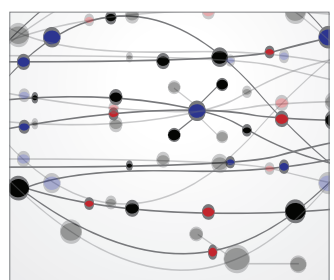

\section{The Scientific} World Journal
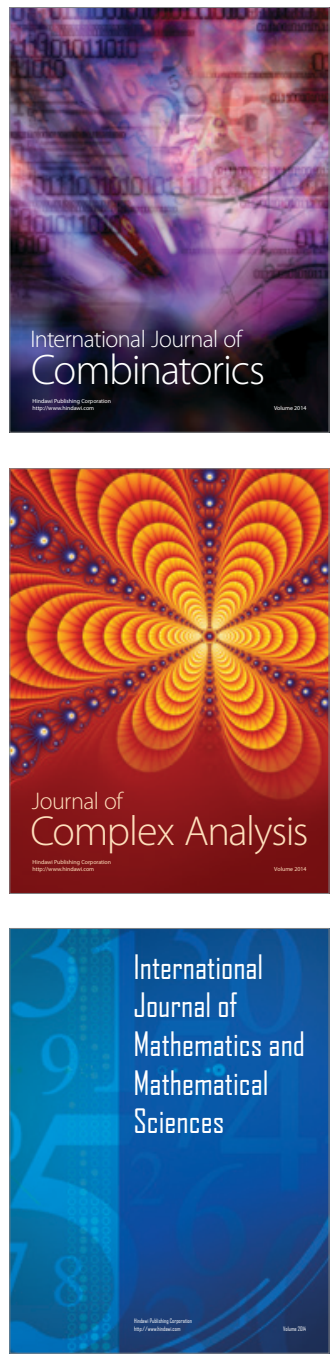
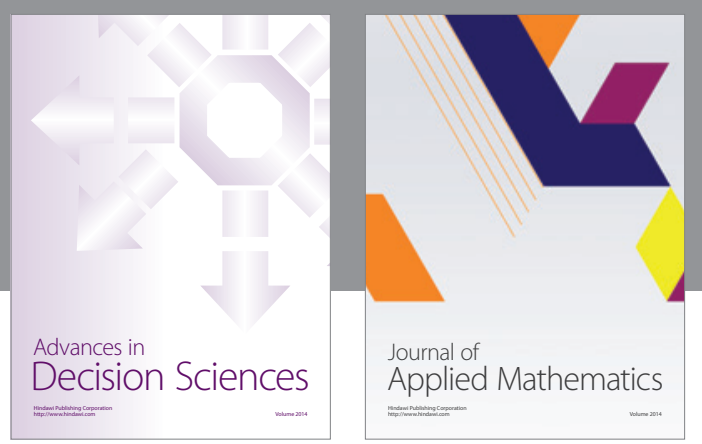

Algebra

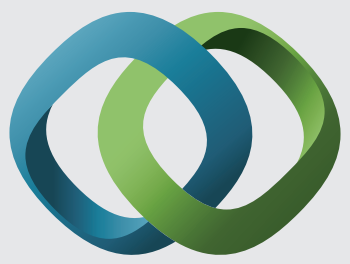

\section{Hindawi}

Submit your manuscripts at

https://www.hindawi.com
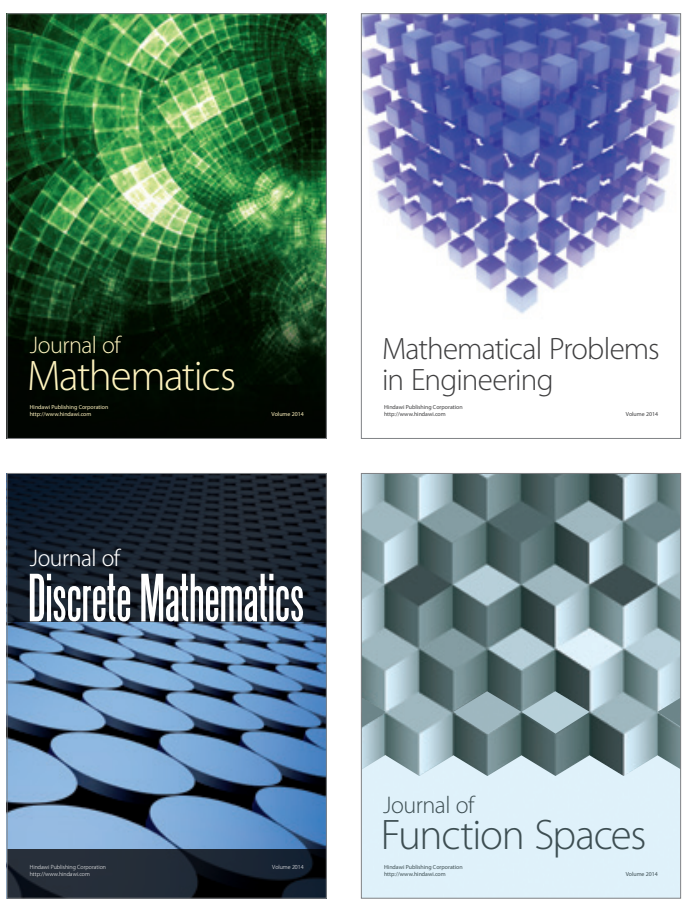

Mathematical Problems in Engineering
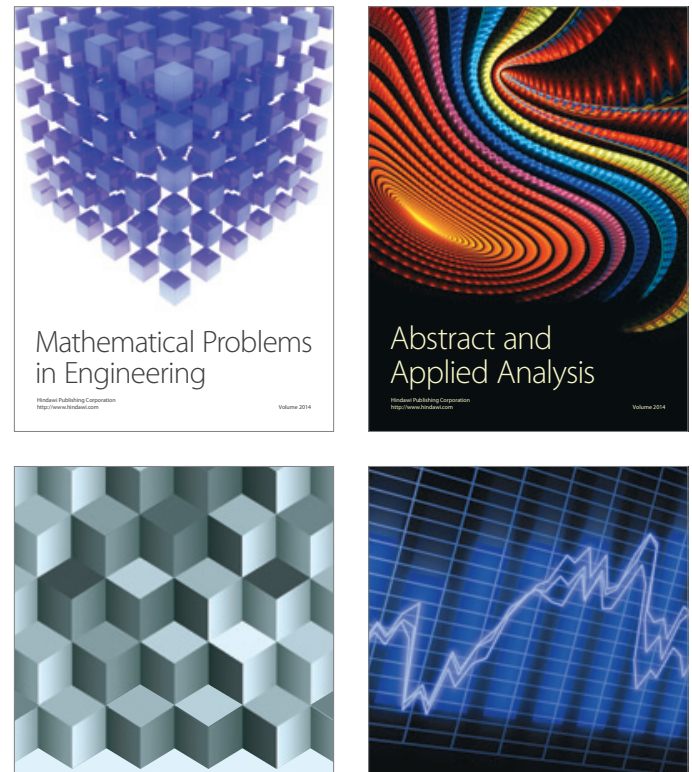

Journal of

Function Spaces

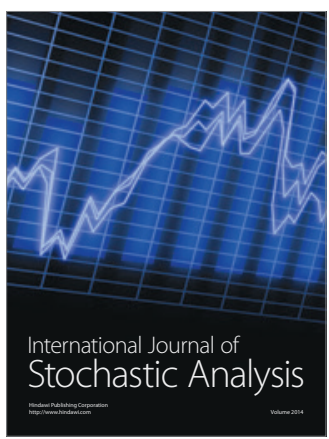

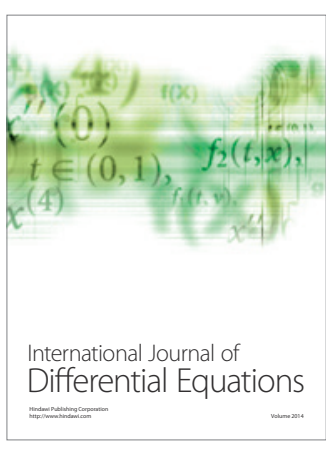
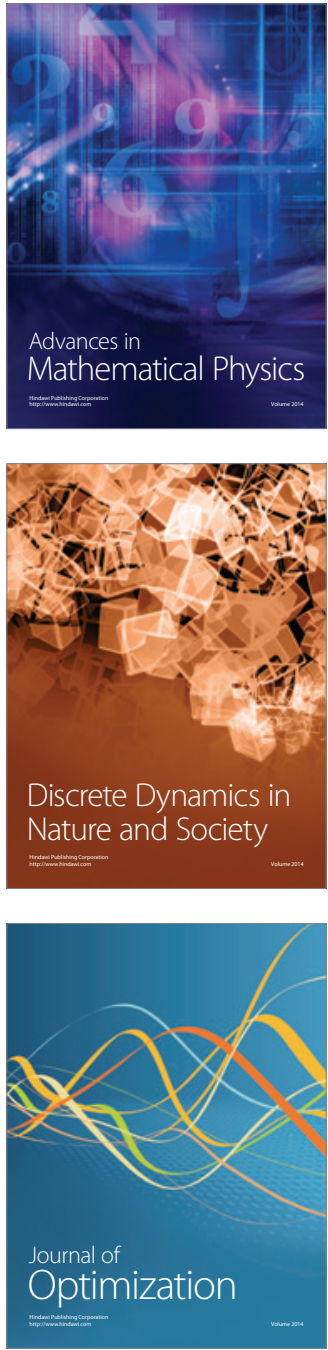